\title{
Lattice Yang-Mills Theory at Nonzero Temperature and the Confinement Problem
}

\author{
Christian Borgs and Erhard Seiler
}

Max-Planck-Institut für Physik und Astrophysik, Werner-Heisenberg-Institut für Physik, D-800 München, Federal Republic of Germany

\begin{abstract}
We discuss finite temperature lattice Yang-Mills theory with special attention to the confinement problem. The relationship between the confinement criteria of Wilson, Polyakov, and 't Hooft is clarified by establishing a string of inequalities between the corresponding string tensions.

The close connection between finite temperature Yang-Mills models and spin models is exploited to obtain new and rather sharp upper bounds for the critical coupling constant above which there is confinement. This same analogy also allows us to establish infrared bounds for the gauge models that yield a lower bound for this critical coupling and thereby show the existence of a weak coupling regime without confinement at nonzero temperature in three or more space dimensions.

Finally we discuss extension of our results to other forms of the lattice action, the Hamiltonian lattice models of Kogut and Susskind and 't Hooft's $N \rightarrow \infty$ limit.
\end{abstract}

\section{Introduction}

One of the outstanding problems of quantum field theory is to understand the socalled confinement of quarks. In the most general sense this means the question why the particle content of quantum chromodynamics (QCD) consists only of hadrons but not of anything like quarks or gluons (not even in bleached form). Since this is an almost intractable problem, at least for the moment, one normally considers some simplified version of it.

First of all, since (continuum) QCD has not been constructed so far, one uses the device of replacing space-time or at least space by a lattice; together with some more or less well-founded scaling hypotheses this still allows one to gain insight into the properties of the continuum theory. On the other hand the well-developed machinery of lattice statistical mechanics becomes available for the analysis.

The next drastic step that is conventionally employed consists of eliminating the quarks from the theory and only considering "quark test charges" or "infinitely 
heavy quark sources" as probes to test the properties of the "glue" that is supposed to keep the quarks together. This leads to the well-known Wilson criterion for confinement [61].

So far even this reduced problem has not been settled completely but both numerical [9] and analytical work $[8,25,34,36,55]$ make it plausible that in four spacetime dimensions nonabelian lattice gauge theories in the usual "compact" form show indeed "permanent confinement of quarks" (attempts to see signs of confinement in "noncompact" lattice versions of QCD have produced negative results $[39,48])$.

The problem of confinement in this restricted sense can also be posed in a slightly different form: one may ask whether the (free) energy per unit length of a chromo-electric flux tube goes to zero if the tube is allowed infinite room in the transverse directions or if it tends to a nonzero limit.

Or one may ask whether the minimal energy of a pair of quark sources grows indefinitely with their separation, or levels off. This last question is most naturally first asked in the framework of quantum field theory at finite temperature with "energy" replaced by "free energy", as Polyakov pointed out some years ago [40].

This is also an interesting question in its own right. QCD at finite temperature is relevant both for the very early universe and possibly for superdense stars; one might argue that even present day experiments do not work at temperature zero but at $2.7 \mathrm{~K}$, the temperature of the cosmic microwave background.

In this paper we first give a systematic discussion of finite temperature lattice Yang-Mills theory (Sect. II), in particular we discuss the introduction of external sources in this framework in some detail. While these concepts have been occurring in the physics literature for some time, we feel that a completely satisfactory derivation of the formulae which are employed is missing so far (this might also be the reason why the usual formulae are not absolutely correct). This is why we include this rather elementary section. We also include a careful treatment of the so-called $\tau$-continuum limit that removes the lattice cutoff in the time direction and leads to the Hamiltonian formulation of lattice gauge theories due to Kogut and Susskind [26]. Some rather tedious technical details of this limit are banished to an appendix.

We then introduce the three concepts of confinement (Wilson, Polyakov, 't Hooft) and prove inequalities between the associated string tensions. It turns out that confinement à la 't Hooft implies all other types of confinement and confinement à la Polyakov implies confinement in Wilson's sense.

We then adapt the formalism of Durhuus and Fröhlich [15] linking YangMills theories in $d+1$ dimensions to spin models in $d$ dimensions to the problem considered here. By combining this formalism with the random walk methods for spin systems of the same authors, we obtain a new and sharper lower bound for a possible critical coupling of a deconfining transition.

Section III is devoted to the proof and application of infrared bounds in the sense of Fröhlich et al. [18] adapted to the case of lattice gauge theories. They lead to a rigorous proof of the existence of a weak coupling regime without confinement at finite temperature in any lattice gauge model in at least four space-time dimensions. This has been expected on the basis of heuristic arguments $[40,41,52$, $60]$ and numerical studies [16, 27, 33]. 
It turns out that the high temperature, weak coupling regime of lattice gauge theories resembles a ferromagnet at low temperature and breakdown of confinement corresponds to the existence of spontaneous magnetization in the ferromagnetic analog.

In Sect. III we first show how deconfinement is proven for the highest possible temperature on the lattice since this is technically easier. After that we treat the general case; our bounds are strong enough to carry over to the $\tau$-continuum limit and to 't Hooft's $N \rightarrow \infty$ limit $\left[g^{2} N\right.$ fixed; $N$ refers to the gauge group $\mathrm{U}(N)$ or $\mathrm{SU}(N)]$.

Finally in Sect. IV we describe various modifications of the model that can also be treated and we discuss some open questions such as Debye screening in the gluon plasma and the behavior of full QCD with dynamical quarks.

Section III is based to a large extent on [2]; a brief description of the results appeared in [3].

\section{Lattice Gauge Theories at Positive Temperature}

We assume that the reader has some familiarity with the general formalism of lattice gauge theories (see for instance [45]).

In the general formalism $[38,45]$ reflection (=physical) positivity is used to construct a quantum mechanical Hilbert space and a transfer matrix ( $\approx$ Hamiltonian) in a rather abstract fashion. The fact that the lattice action couples only neighboring sites or links (essentially a Markov property) then allows us to go to a simpler and more explicit description of the Hilbert space and the transfer matrix which is more suitable for our investigation. We start with this description and leave it to the reader to establish the almost obvious relation to the general formalism. Everything is formulated in a finite volume; the thermodynamic limit will be taken when needed.

\section{Hilbert Space}

We consider a spatial box $\Lambda_{0} \subset \mathbb{Z}^{d}$. We may think of this as the time zero slice of a space-time lattice $\Lambda_{0} \times \tau \mathbb{Z}$. We pick a positive orientation for all $p$-cells (sites, links, plaquettes etc.) of $\Lambda_{0}$ and $\Lambda_{0} \times \tau \mathbb{Z}$.

The classical configuration space consists of all maps from the positively oriented nearest neighbor links $\langle x y\rangle \in \Lambda_{0}$ into a compact group $G$, the gauge group [which in most applications will be $U(N)$ or $\mathrm{SU}(N)$ ]. If $\langle x y\rangle$ is positively oriented we define $g_{y x} \equiv g_{x y}^{-1}$. Our Hilbert space $\mathscr{H}_{A_{0}}$ is simply the $L^{2}$ space over this configuration space with respect to Haar measure:

$$
\mathscr{H}_{\Lambda_{0}} \equiv L^{2}\left(\left\{g_{x y}\right\}\right) \equiv L^{2}(g) \text {. }
$$

\section{Transfer Matrix}

The transfer matrix $\mathscr{T}$ is a positive trace class operator on $\mathscr{H}_{\Lambda_{0}}$, which sometimes will be written as $\mathscr{T}=\exp (-\tau H)$ and corresponds to translation by one lattice unit $\tau$ in the time direction. (The general formalism provides such a $\mathscr{T}$ if the model possesses reflection positivity with respect to reflection both in lattice planes and 
in planes lying half-way between lattice planes.) $\mathscr{T}$ is of the form

$$
\mathscr{T}=M T_{0} M,
$$

where $M$ is multiplication by a positive function and $T_{0}$ is a positive convolution operator. The precise form of $M$ and $T_{0}$ depends on the version of lattice gauge theory considered.

For instance for the lattice gauge theory of (Wegner [59] and) Wilson [61]

$$
M=M_{W}\left(\left\{g_{x y}\right\}\right)=\exp \frac{1}{2} J_{M} \sum_{P} \operatorname{Re}\left(\chi\left(g_{\partial P}\right)-\chi(\mathbb{1})\right),
$$

where the sum is over all positively oriented plaquettes of $\Lambda_{0}$ and $\chi$ is a faithful character of $G ; g_{\partial P}$ stands as usual for the ordered product $\prod_{\langle x y\rangle \in \partial P} g_{x y}$ (the starting point is arbitrary). Furthermore $T_{0}$ is given by convolution with

$$
T_{W}(\mathbf{g})=\exp \left\{J_{E} \sum_{\langle x y\rangle} \operatorname{Re}\left(\chi\left(g_{x y}\right)-\chi(\mathbb{1})\right)\right\},
$$

where the sum is now over all positively oriented links. (The subscripts $E$ and $M$ refer to "electric" versus "magnetic".)

Contact with the usual euclidean formulation is made by setting

$$
J_{M}=\frac{2 \tau}{\varepsilon} \frac{\varepsilon^{d-3}}{g^{2}}, \quad J_{E}=\frac{2 \varepsilon}{\tau} \frac{\varepsilon^{d-3}}{g^{2}} .
$$

[For $G=\mathrm{U}(1)$ one conventionally replaces $2 / g^{2}$ by $1 / e^{2}$.] To sum up: The Wilson transfer matrix has the kernel

$$
\begin{aligned}
\mathscr{T}_{W}(\mathbf{g}, \mathbf{h})= & \exp \left\{J_{E} \sum_{\langle x y\rangle} \operatorname{Re}\left(\chi\left(g_{x y} h_{x y}^{-1}\right)-\chi(\mathbb{1})\right)\right\} \\
& \cdot \exp \left\{\frac{1}{2} J_{M} \sum_{P} \operatorname{Re}\left(\chi\left(g_{\partial P}\right)+\chi\left(h_{\partial P}\right)-2 \chi(\mathbb{1})\right\} .\right.
\end{aligned}
$$

Our notation is motivated by the $\tau$-continuum limit. Sending $\tau$ to zero we obtain as will be seen below:

$$
H=\lim _{\tau \rightarrow 0} \frac{1}{\tau}\left(\mathbb{1}-\mathscr{T}_{W}\right)=-\frac{2}{g^{2}} \sum_{P} \varepsilon^{d-4} \operatorname{Re}\left(\chi\left(g_{\partial P}\right)-\chi(\mathbb{1})\right)-\frac{g^{2}}{2} \sum_{\langle x y\rangle} \varepsilon^{2-d} \Delta_{x y},
$$

where $\Delta_{x y}$ is the Laplace-Beltrami operator on $G$ and corresponds to $E^{2}$ (the square of the electric field) whereas the first term corresponds to $B^{2}$ (the square of the magnetic field in the classical continuum Hamiltonian $\frac{1}{2} \int\left(E^{2}+B^{2}\right)$.)

Other lattice gauge models have different functions $M$ and $T_{0}$; for us it is essential that

(1) $M$ and $T_{0}$ are positive definite, positive and

(2) for $g^{2} \rightarrow 0$, become concentrated more and more at the unit element of the group. One other choice that is of interest is the so-called heat kernel or Villain action:

$$
\begin{aligned}
& M^{2}=M_{H}^{2}(\underset{\sim}{g})=\prod_{P}\left(\exp J_{M}^{-1} \Delta\right)\left(g_{\partial P}\right), \\
& T_{0}=T_{H}(\underset{\sim}{g})=\prod_{\langle x y\rangle}\left(\exp J_{E}^{-1} \Delta\right)\left(g_{\langle x y\rangle}\right) .
\end{aligned}
$$


But it is also possible to make different choices for $T_{0}$ and $M$, for instance to choose $T=T_{H}$ and $M=M_{W}$. A simple fact is the following

Lemma II.1. Let $T_{0}=T_{H}$ or $T_{W}, M=M_{H}$ or $M_{W}$. Then the transfer matrix is bounded and strictly positive.

Proof. Only $\mathscr{T}>0$ is not completely trivial (see [29] for a proof for a special case). Since obviously $M>0$ and $T_{H}>0$, we only have to show $T_{W}>0$. Since $T_{W}$ is a tensor product over links, it suffices to show that the operator $t$ given by convolution with the function

$$
t(g)=\exp J \operatorname{Re} \chi(g)
$$

is strictly positive.

Using the Peter-Weyl theorem [24] one gets for the eigenvalues of $t$

$$
\begin{aligned}
m_{\tau} & =\frac{1}{\chi_{\tau}(\mathbb{1})} \int d g \overline{\chi_{\tau}(g)} \exp (J \operatorname{Re} \chi(g)) \\
& =\frac{1}{\chi_{\tau}(\mathbb{1})} \sum_{n, m \geqq 0}\left(\frac{J}{2}\right)^{n+m} \frac{1}{n ! m !} c_{n m}^{\tau},
\end{aligned}
$$

with

$$
c_{n m}^{\tau}=\int d g \overline{\chi_{\tau}(g)} \chi(g)^{n} \overline{\chi(g)^{m}} \geqq 0 .
$$

$\tau$ is a label for the irreductible representations of $G$. Since $\chi$ was assumed to be faithful, $\operatorname{Re} \chi(g)<\chi(\mathbb{1})$ if $g \neq 1$ and for $J \rightarrow \infty, \exp J \operatorname{Re} \chi(g)$ becomes very sharply peaked around $\mathbb{1}$. Therefore

$$
\frac{m_{\tau}}{m_{0}} \rightarrow 1 \quad \text { as } \quad J \rightarrow \infty,
$$

and thus for each $\tau$ at least one $c_{n m}^{\tau}>0$ which implies $m_{\tau}>0$ for all $J>0$.

Remark. Lüscher [29] proved $\mathscr{T}>0$ for the case $G=\mathrm{U}(1)$ or $\mathrm{SU}(N)$.

The lemma allows us to define a self-adjoint semi-bounded Hamiltonian $H_{\tau}$ by

$$
H_{\tau}=-\frac{1}{\tau} \log \mathscr{T} \text {. }
$$

Theorem II.2. Let $G$ be a compact Lie group

$$
V=-\frac{1}{2 \tau} \log M,
$$

uniformly Lipschitz continuous and

$$
H=-\varepsilon^{2-d} \sum_{\langle x y\rangle} \frac{g^{2}}{2} \Delta_{x y}+V .
$$

Then for $T_{0}=T_{H}$ or $T_{W}$

$$
\mathscr{T}^{\beta / \tau}=e^{-\beta H_{\tau}} \underset{\tau \rightarrow \sigma}{\longrightarrow} e^{-\beta H}
$$


in all $J_{p}$ norms $(p \geqq 1)\left(J_{p}\right.$ is the space of all compact operators $A$ such that

$$
\left.\|A\|_{p} \equiv\left(\operatorname{Tr}\left(A^{*} A\right)^{p / 2}\right)^{1 / p}<\infty\right) .
$$

Proof. This requires some rather technical considerations which we describe in Appendix A.

Remarks. 1. Strong convergence has been proven by Gawedzki [19]. See also [10].

2. Note that the theorem involves a choice of normalization for the generators $i L^{a}$ of the group $G$ which is given by

$$
\chi\left(L^{a} L^{b}\right)=\frac{1}{2} \delta_{a b}
$$

In the abelian case one normally drops the factor $\frac{1}{2}$ which leads to a change from $g^{2} / 2$ to $g^{2} / 4$ in the Hamiltonian; with $g^{2}=2 e^{2}$ we obtain again the standard form, but with $e^{2}$ in place of $g^{2}$.

\section{External Charges, Gauss's Law, Confinement}

$\mathscr{H}_{\Lambda_{0}}$ is not gauge invariant. If we transform

$$
\left\{g_{x y}\right\} \mapsto\left\{g_{x y}\right\}^{h} \equiv\left\{h_{x}^{-1} g_{x y} h_{y}\right\}
$$

(where $\underset{\sim}{h}$ is a function from $\Lambda_{0}$ into $G$ ) this induces a unitary map $\mathrm{U}(h)$ on $\mathscr{H}_{\Lambda_{0}}$ :

$$
(\mathrm{U}(\underset{\sim}{h}) \Psi)\left(\left\{g_{x y}\right\}\right)=\Psi\left(\left\{g_{x y}\right\}^{h}\right) .
$$

$\mathrm{U}(\underset{\mathrm{h}}{\mathrm{h}})$ defines a unitary representation of $\mathscr{G}=\chi_{x \in \Lambda_{0}} G$ on $\mathscr{H}_{\Lambda_{0}}$; we may decompose $\mathscr{H}_{\Lambda_{0}}$ according to the irreducible representations $\left\{\tau_{x}\right\}_{x \in \Lambda_{0}}$ of $\mathscr{G}$ :

$$
\mathscr{H}_{\Lambda_{0}}=\bigoplus \mathscr{H}_{\left\{\tau_{x}\right\}} .
$$

The projection on $\mathscr{H}_{\left\{\tau_{x}\right\}}$ we denote by

$$
P_{\left\{\tau_{x}\right\}}=\prod_{x \in \Lambda_{0}} P_{\tau_{x}}
$$

It is easy to see by the Peter-Weyl theorem [24] that

$$
\left(P_{\tau_{x_{0}}} \Psi\right)\left(\left\{g_{x y}\right\}\right)=d_{\tau_{x_{0}}} \int \overline{\chi_{\tau_{x_{0}}}(h)} \Psi\left(\left\{g_{x y}\right\}^{h_{x_{0}}}\right) d h
$$

where ${\underset{\sim}{x_{0}}}_{\text {in }}$ is the map

$$
x \mapsto h_{x}=\left\{\begin{array}{ll}
\mathbb{1} & \left(x \neq x_{0}\right) \\
h & \left(x=x_{0}\right)
\end{array} .\right.
$$

and $d_{\tau_{x_{0}}}$ is the dimension of $\tau_{x_{0}}$ (cf. [45]).

We say that $\mathscr{H}_{\left\{\tau_{x}\right\}}$ is the subspace having (external) charges $\left\{\tau_{x}\right\}$; if $\tau_{x}=\mathbb{1}$ (the trivial representation), we say that there is no charge at $x$.

Clearly

$$
\left[\mathscr{T}, P_{\left\{\tau_{x}\right\}}\right]=0
$$

etc., so these charges do not move; for that reason they are called infinitely heavy. The subspaces $\mathscr{H}_{\left\{\tau_{x}\right\}}$ may be considered as different superselection sectors. 
There is a slightly different way of looking at these sectors based on a lattice version of Gauss's law: Let $G$ now be a compact Lie group, assume that for each link a direction has been selected, choose a basis $\left\{L_{a}\right\}_{a=1}^{n}$ of $\mathfrak{g}$, the Lie algebra of $G$. Then we define (in a slightly arbitrary fashion) the operator of the electric field $E_{x_{0} y_{0}}^{a}$ by

$$
\left(\left(E_{x_{0} y_{0}}^{a}\right) \Psi\right)(\underset{\sim}{g})=\lim _{\varepsilon \rightarrow 0} \frac{1}{\varepsilon}\left(\Psi\left(g_{\varepsilon}\right)-\Psi(\underset{g}{g})\right)
$$

where

$$
\left(g_{\varepsilon}\right)_{x y}= \begin{cases}g_{x y} & \left(\langle x y\rangle \neq\left\langle x_{0} y_{0}\right\rangle\right) \\ g_{x_{0} y_{0}} e^{\varepsilon L_{a}} & \left(\langle x y\rangle=\left\langle x_{0} y_{0}\right\rangle\right) .\end{cases}
$$

Furthermore let $\varrho_{x_{0}}^{a}$ be defined as the operator of an infinitesimal gauge transformation at $x_{0}$ :

$$
\left(\varrho_{x_{0}}^{a} \Psi\right)(g)=\lim _{\varepsilon \rightarrow \sigma} \frac{1}{\varepsilon}\left(\Psi(\underbrace{g^{h_{\varepsilon}}})-\Psi(\underset{\sim}{g})\right)
$$

where now

$$
\left(h_{\varepsilon}\right)_{x}= \begin{cases}\mathbb{1} & \left(x \neq x_{0}\right) \\ \exp \varepsilon L_{0} & \left(x=x_{0}\right)\end{cases}
$$

It can then be checked that

$$
(D \cdot E)_{x_{0}}^{a}=\varrho_{x_{0}}^{a}
$$

where $(D \cdot E)_{x_{0}}$ is some kind of covariant divergence of $E$ at $x_{0}$ :

$$
(D \cdot E)_{x_{0}}^{a}=\sum_{\langle x y\rangle: x=x_{0}} E_{x y}^{a}-\sum_{\langle x y\rangle: y=x_{0}}\left(g_{x y}^{-1} E_{x y} g_{x y}\right)^{a}
$$

in obvious notation.

By Stone's theorem $\varrho_{x_{0}}^{a}$ is an antiselfadjoint operator; the set $\left\{\varrho_{x_{0}}^{a}\right\}_{a=1}^{n}$ spans a representation of the Lie algebra $g$ of $G$ and the decomposition (II.11) corresponds to the decomposition of this representation into irreducible components.

To say that at point $x$ there is an external charge $\tau_{x}$ means therefore that the "charge densities" $\varrho_{x}^{a}$ act according to the irreducible representation $\tau_{x}$. This is the appropriate generalization of the statement (that makes sense only for abelian $G$ ) that $\varrho_{x}$ has eigenvalue $\tau_{x}$.

We can now define partition functions and free energies corresponding to inverse temperature $\beta$ and external charges $\left\{\tau_{x}\right\}$ by

$$
Z_{\left\{\tau_{x}\right\}} \equiv e^{-\beta F_{\left\{\tau_{x}\right\}}} \equiv \operatorname{Tr} P_{\left\{\tau_{x}\right\}} e^{-\beta H_{\tau}}=\operatorname{Tr} P_{\left\{\tau_{x}\right\}} \mathscr{T}^{\beta / \tau}
$$

At this point we can make contact with the usual euclidean lattice formulation of gauge theories. There one starts with an action

$$
S=\sum_{P} S_{P}\left(g_{\partial P}\right)
$$


where the sum is over all plaquettes in a space-time lattice $\tilde{\Lambda}$ which we assume to be of the form

$$
\tilde{\Lambda}=\Lambda_{0} \times\left\{\tau, 2 \tau, \ldots, \beta=L_{0} \tau\right\}
$$

We impose periodic boundary conditions in time, i.e. we identify $\beta+t$ with $t$. For Wilson's model we specify furthermore $S_{W}=\sum_{P} S_{P, W}$ with

$$
-S_{P, W}=\left\{\begin{array}{l}
J_{M} \operatorname{Re} \chi\left(g_{\partial P}\right): P \text { spatial } \\
J_{E} \operatorname{Re} \chi\left(g_{\partial P}\right): P \text { contains the time direction, }
\end{array}\right.
$$

where $\chi$ is as before a faithful character of $G$. Then we have

\section{Lemma II.3.}

$$
\int e^{-S_{W}} d g=Z_{0}=\operatorname{Tr} P_{0} \mathscr{T}^{\beta / \tau},
$$

where $P_{0}$ is the projection operator onto the chargeless subspace.

Proof (cf. also [7] for a special case). This is just a computation. Gauge invariance may be used to freeze all links in time direction except in one time layer in $\int e^{-S_{W}} d g$. These remaining link variables may be identified with the gauge variables $\underset{\sim}{h}$ in the definition of $P_{0}$ (recall that

$$
\left(P_{0} \mathscr{T}\right)\left(\left\{g_{x y}\right\},\left\{g_{x y}^{\prime}\right\}\right)=\int \prod d h_{x} \mathscr{T}\left(\left\{h_{x}^{-1} g_{x y} h_{y}\right\},\left\{g_{x y}^{\prime}\right\}\right) \text {. }
$$

Lemma I1.4. Let $L_{x}$ denote a closed loop in time direction (closed because of the periodic boundary conditions), $g_{L_{x}}$ the corresponding ordered product of link variables (with arbitrary starting point), $\chi_{\tau_{x}}$ the character of the representation $\tau_{x}$. Then

$$
\int e^{-S_{W}} \prod_{i=1}^{k}\left(d_{\tau_{x_{i}}} \chi_{\tau_{x_{i}}}\left(g_{L_{x_{i}}}\right)\right)=Z_{\tau_{x_{1} \ldots} \ldots \tau_{x_{k}}} \equiv \operatorname{Tr} P_{\left\{\tau_{x}\right\}} \mathscr{T}_{W}^{\beta / \tau},
$$

where $\tau_{x_{i}}=\tau_{x}$ if $x=x_{i}$ and $\tau_{x}=\mathbb{1}$ otherwise.

Proof. This is almost the same computation as before.

Remarks. 1. $\chi_{\tau_{x}}\left(g_{L_{x}}\right)$ is variously called a Polyakov loop, thermal Wilson loop, Wilson line, etc.... Its relevance was first pointed out by Polyakov [40].

2. The formulae hold just as well for other actions than Wilson's.

3. $Z_{\left\{\tau_{x}\right\}}$ and $F_{\left\{\tau_{x}\right\}}=-\frac{1}{\beta} \log Z_{\left\{\tau_{x}\right\}}$ have a finite limit as $\tau \rightarrow 0$;

$\lim _{\tau \rightarrow \sigma} Z_{\left\{\tau_{x}\right\}}=\operatorname{Tr} P_{\left\{\tau_{x}\right\}} e^{-\beta H}$. This is an obvious consequence of Theorem II.2.

These facts suggest the following definition: Let

$$
\begin{aligned}
F_{0}^{\beta} & \equiv-\frac{1}{\beta} \log Z_{0}, \\
F_{q(x) \bar{q}(y)}^{\beta} & \equiv-\frac{1}{\beta} \log Z_{q(x) \bar{q}(y)}
\end{aligned}
$$


be the free energies with and without external quark sources $[q$ stands for the fundamental representation of $G=\mathrm{SU}(N)$ or $\mathrm{U}(N)] ; \bar{q}$ is its conjugate representation. by

We then define a (temperature dependent) quark-antiquark potential $V_{q(x) \bar{q}(y)}^{\beta}$

$$
V_{q(x) \bar{q}(y)}^{\beta} \equiv V_{q \bar{q}}(x-y)=F_{q(x) \bar{q}(y)}^{\beta}-F_{0}^{\beta}=-\frac{1}{\beta} \log \left(G(x-y) d_{q}^{2}\right),
$$

where

$$
G(x-y)=\frac{1}{Z_{0}} \int e^{-s} \overline{\chi_{q}\left(g_{L_{x}}\right)} \chi_{q}\left(g_{L_{y}}\right)
$$

(we assumed translation invariance, as guaranteed for instance by periodic b.c. in writing $G$ as a function of $x-y$ ). (Linear) confinement is then understood to mean that in the thermodynamic limit $\left.\Lambda_{0}\right\rceil \varepsilon \mathbb{Z}^{d}$

$$
\lim _{|x-y| \rightarrow \infty} \frac{1}{|x-y|} V_{q \bar{q}}(x-y)>0,
$$

which is equivalent to exponential decay of $G(x-y)$, whereas long range order of $G$ [i.e. $\lim G(x) \neq 0$ ] clearly means absence of confinement.

Remark. This way of looking at the confinement problem is due to Polyakov [40]. We mention some simple general properties of $V_{q \bar{q}}$ :

Lemma II.5 (cf. [44]). Assume that the thermodynamic limit has been taken at least in the 1-direction, and let $\hat{e}_{1}$ be the unit vector in that direction. Then $V_{q \bar{q}}\left(\right.$ Le $\left.\hat{e}_{1}\right)$ grows with $L$, is concave and $\frac{V_{q \bar{q}}\left(L \hat{e}_{1}\right)-V_{q \bar{q}}(0)}{L}$ falls with $L$.

Remark. This says that the quark-antiquark potential is always attractive but the force decreases with growing distance.

Proof. The first two statements follow from the existence of a positive transfer matrix $\mathscr{T}$ for translations in the 1 -direction and the spectral theorem:

$$
G\left(L \hat{e}_{1}\right)=\int d \mu(\omega) e^{-\omega L},
$$

with some positive measure $d \mu$ because we can express $G\left(L \hat{e}_{1}\right)$ as $\left(\Psi, \mathscr{T}^{L} \Psi\right)$. The last statement is an easy consequence of the first two.

There is a slightly different way of looking at the confinement problem that consists of studying the free energy of an electric flux tube impressed on a periodic box; this concept is due to 't Hooft [54].

We want to describe it here from our point of view and establish some connections to the previously defined concept of confinement. The following discussion uses some elementary notions and facts of lattice (co)-homology: we refer to [58] or the excellent review [14] for details.

Let $\omega$ be an element of $C(G)$, the center of $G$. We define the operator of the "central electric field" $E_{x_{0} y_{0}}^{\omega}$ by

$$
\left(E_{x_{0} y_{0}}^{\omega} \Psi\right)(\underset{\sim}{g}) \equiv \Psi(\underset{\sim}{g} \omega),
$$


where

$$
(\underset{\sim}{\omega})_{x y}= \begin{cases}\mathbb{1} & \left(\left\langle x_{0} y_{0}\right\rangle \neq\langle x y\rangle \neq\left\langle y_{0} x_{0}\right\rangle\right) \\ \omega & \left(\langle x y\rangle=\left\langle x_{0} y_{0}\right\rangle\right) \\ \omega^{-1} & \left(\langle x y\rangle=\left\langle y_{0} x_{0}\right\rangle\right)\end{cases}
$$

Because this "electric field" is now abelian, it allows us to define a "central electric flux" $\Phi^{\omega}(\Sigma)$ through a closed 2-surface $* \Sigma$ on the dual lattice. More generally let $\Sigma$ be a 1 co-cycle on the original lattice, that means $\Sigma$ is a function from the oriented links $\langle x y\rangle$ into the integers obeying

$$
\Sigma(\langle y x\rangle)=-\Sigma(\langle x y\rangle)
$$

and

$$
\sum_{\langle x y\rangle \in \partial p} \sum(\langle x y\rangle)=0
$$

where the sum is over all links $\langle x y\rangle$ contained in the boundary $\partial p$ of a plaquette $p$ with positive orientation. Then

$$
\Phi^{\omega}(\Sigma) \equiv \prod_{\langle x y\rangle}^{\prime}\left(E_{x y}^{\omega}\right)^{\Sigma(\langle x y\rangle)}
$$

where the product $\prod^{\prime}$ is over all positively oriented links.

A "central charge" $\varrho_{x_{0}}^{\omega}$ at a point $x_{0}$ may then be defined by the central electric flux through the elementary cube of the dual lattice enclosing $x_{0}$, i.e.

$$
\varrho_{x_{0}}^{\omega} \equiv \prod_{\substack{\langle x y\rangle \\ x=x_{0}}} E_{x y}^{\omega} .
$$

It is then clear that the spectrum of $E_{x y}^{\omega}, \Phi^{\omega}(\Sigma), \varrho_{x_{0}}^{\omega}$ lies on the unit circle (because all these operators are unitary); furthermore it is discrete : $E_{x y}, \Phi^{\omega}(\Sigma), \varrho_{x_{0}}^{\omega}$ have the possible eigenvalues $\frac{\chi_{\tau}(\omega)}{\chi_{\tau}(\mathbb{1})}=\tilde{\chi}_{\tilde{\tau}}(\omega)$, where $\tilde{\tau}$ is the irreducible representation of $C(G)$ induced by the representation $\tau$ of $G$ and $\tilde{\chi}_{\tilde{\tau}}$ is the corresponding character of $C(G)$. This means that the eigenvalues of $E_{x y}^{\omega}, \Phi^{\omega}(\Sigma), \varrho_{x_{0}}^{\omega}$ will lie in $\hat{C}(G)$, the dual of the center of $G$.

Note that $E_{x y}^{\omega}, \varrho_{x_{0}}^{\omega}, \Phi^{\omega}(\Sigma)$ commute with all gauge transformations and with the convolution part of the transfer matrix. $\varrho_{x_{0}}^{\omega}$ commutes with the full transfer matrix because it is a gauge transformation. Furthermore

$$
\left[\mathscr{T}, \Phi^{\omega}(\Sigma)\right]=0 .
$$

because the multiplication operator $M$ is only a function of $g_{\partial P}$ and $\Sigma$ is coclosed [Eq. (II.26)].

There is also a Stokes' formula: Let $* \Omega$ be a set of elementary cubes of the dual lattice (or more generally $\Omega$ a 0 -cochain of the original lattice) and $* \Sigma=\partial * \Omega$ its boundary (properly oriented), i.e. $\Sigma$ its coboundary. Then obviously

$$
\Phi^{\omega}(\Sigma)=\prod_{x \in * \Omega}^{\prime} \varrho_{x}^{\omega}
$$


(again the product $\Pi^{\prime}$ goes only over the $x$ contained in $\Omega$ with positive orientation) or more generally

$$
\Phi^{\omega}(\Sigma)=\prod_{x}^{\prime}\left(\varrho_{x}^{\omega}\right)^{\Omega(x)}
$$

Let now $\chi_{\tau} \in \hat{C}(G)$. The corresponding eigenprojection $Q^{\tau}(\Sigma)$ for the electric flux through $\Sigma$ is then

$$
Q^{\tau}(\Sigma)=\int_{C(G)} \Phi^{\omega}(\Sigma) \overline{\chi_{\tau}(\omega)} d \omega,
$$

where $d \omega$ is the normalized Haar measure on $C(G)$.

We are now ready to define the relative free energy of central electric flux in a periodic box $\Lambda_{0}$ of lengths $L_{1}, L_{2}, L_{3}$ by

$$
\Delta F^{\tau}(23)=-\frac{1}{\beta}\left\{\log \operatorname{Tr} P_{0} Q^{\tau}\left(\Sigma_{23}\right) e^{-\beta H}-\log \operatorname{Tr} P_{0} Q^{\mathbb{1}}\left(\Sigma_{23}\right) e^{-\beta H}\right\},
$$

where $\Sigma_{23}$ is a direct product of fundamental 1-cycles in the 2-and 3-directions of $\Lambda_{0}$ and $Q^{\mathbb{1}}$ is the projection corresponding to the trivial representation. Note that $P_{0}$ and $Q^{\tau}$ commute.

By (II.29) $\Sigma_{23}$ may be deformed into a homotopic 2-cycle without changing anything, because there are no external charges around $\left(P_{0} \varrho_{x_{0}}^{\omega}=P_{0}\right.$ because $\varrho_{x_{0}}^{\omega}$ is a gauge transformation).

Let now

$$
Z_{\beta L_{1} ; L_{2} L_{3}}^{\tau}=\operatorname{Tr} P_{0} Q^{\tau}\left(\Sigma_{23}\right) e^{-\beta H}
$$

be the partition function in the presence of electric flux. It has an important symmetry property:

\section{Lemma II.6.}

$$
Z_{\beta L_{1} ; L_{2} L_{3}}^{\tau}=Z_{L_{1} \beta ; L_{2} L_{3}}^{\tau}=Z_{\beta L_{1} ; L_{3} L_{2}}^{\tau}
$$

Proof. The second equality is obvious. To see the first one note that $\operatorname{Tr} \Phi^{\omega}\left(\Sigma_{23}\right) P_{0} e^{-\beta H}$ is obtained from

$$
\operatorname{Tr} P_{0} e^{-\beta H}=\operatorname{Tr} \Phi^{\mathbb{1}}\left(\Sigma_{23}\right) P_{0} e^{-\beta H}
$$

by replacing $S_{p}$ by $\chi(\omega) \chi(\mathbb{1})^{-1} S_{P}$ for one plaquette in each 01 -plane. This is clearly symmetric under interchange of the 0 - and 1-directions.

Next we note some commutation relations:

Lemma II.7. Let $C$ be a closed loop in $\Lambda_{0}, g_{C}$ the corresponding ordered product of link variables ("Wilson loop"). Then

$$
\Phi^{\omega}(\Sigma) \chi_{\tau}\left(g_{C}\right)=\left(\frac{\chi_{\tau}(\omega)}{\chi_{\tau}(\mathbb{1})}\right)^{\Sigma(C)} \chi_{\tau}\left(g_{C}\right) \Phi^{\omega}(\Sigma),
$$

where $\Sigma(C)$ is the 1-cocycle $\Sigma$ evaluated on the 1-cycle $C$. Furthermore

$$
Q^{\sigma}(\Sigma) \chi_{\tau}\left(g_{C}\right)=\chi_{\tau}\left(g_{C}\right) Q^{\tau^{-\Sigma(C)} \sigma}(\Sigma)
$$


where we used the label $\tau$ both for an irreducible representation of $G$ and the element of $\hat{C}(G)$ induced by it.

Remark. (II.35) can be stated verbally as follows:

"Wilson loops create electric flux."

The proof of Lemma II.6 is an elementary computation.

Finally we want to prove some inequalities between the various quantities introduced:

Lemma II.8. Let $\hat{e}_{1}$ be the unit lattice vector in 1-direction and $y=x+L_{1} \varepsilon \hat{e}_{1}$. Then $F_{q(x) \bar{q}(y)}^{\beta}$ is increasing in $\beta$, that is

$$
F_{q(x) \bar{q}(y)}^{\beta} \leqq F_{q(x) \bar{q}(y)}^{\beta^{\prime}},
$$

if $\beta \leqq \beta^{\prime}$ and

$$
G(x-y) \lim _{L \rightarrow \infty}\left(Z_{\beta L ; L_{2} L_{3}}^{\mathbb{1}}\right)^{L_{1} / L} \leqq d_{q}^{2} Z_{\beta L_{1} ; L_{2} L_{3}}^{q}
$$

where $G$ is to be taken in a box that is infinite in the 1-direction and periodic of lengths $L_{2}, L_{3}$ in the 2- and 3-directions.

Proof. (II.36) follows from the definition of the free energy and the standard fact that for $A \geqq 0\left(\operatorname{Tr} A^{t}\right)^{1 / t}$ is decreasing in $t$.

To see (II.37) we have to interchange the rôles of the 0 - and 1-directions. The right hand side is clearly symmetric in $\beta$ and $L_{1}$ (by Lemma II.6). The left hand side can be rewritten as

$$
\lim _{L \rightarrow \infty}\left(\operatorname{Tr} P_{0} \mathscr{T}^{L-L_{1}} M^{*} \mathscr{T}^{L_{1}} M\right)\left(\operatorname{Tr} P_{0} \mathscr{T}^{L}\right)^{-1+L_{1} / L},
$$

where $\mathscr{T}$ is now the transfer matrix in 1-direction and $M$ is the multiplication operator corresponding to the insertion of $\chi\left(g_{L}\right), M^{*}$ its adjoint.

Denote by $e^{-\varepsilon E_{0}}=\left\|\mathscr{T} P_{0}\right\|$ the largest eigenvalue of $\mathscr{T} P_{0}$ and by $\Omega$ the corresponding eigenvector (which is unique by the Perron-Frobenius theorem [43]). Then (II.38) becomes

$$
\left(\Omega, M^{*} \mathscr{T}^{L_{1}} M \Omega\right)
$$

By the Perron-Frobenius theorem $\Omega$ may be chosen to be a positive function in $L^{2}$ (see [43]) and therefore it does not contain electric flux:

$$
Q^{\mathbb{1}}\left(\Sigma_{23}\right) \Omega=\Omega
$$

[because $Q^{\tau}\left(\Sigma_{23}\right)$ commutes with $\mathscr{T}, \Omega$ has to be an eigenvector of it, but because of varying phases the eigenvalue cannot be 1 for $\tau \neq \mathbb{1}$, hence it has to be zero].

Thus by (II.35)

$$
Q^{q}\left(\Sigma_{23}\right) M \Omega=M \Omega
$$

and

$$
\begin{aligned}
\left(\Omega, M^{*} \mathscr{T}^{L_{1}} M \Omega\right) & \leqq \operatorname{Tr}\left(P_{0} Q^{q}\left(\Sigma_{23}\right) \mathscr{T}^{L_{1}}\right)\|M\|_{\infty}^{2} \\
& =Z_{\beta L_{1} ; L_{2} L_{3}}^{q} d_{q}^{2},
\end{aligned}
$$


where in the last step we used the symmetry (Lemma II.6) once more. This proves (II.37).

If we rewrite (II.37) as follows

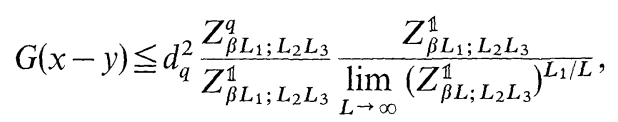

we can look at the behavior for $|x-y|=L_{1} \rightarrow \infty$. The second factor on the right hand side of (II.43) goes to 1 and we obtain

$$
-\lim _{|x-y| \rightarrow \infty} \frac{1}{|x-y|} \log G(x-y) \geqq \lim _{L_{1} \rightarrow \infty} \frac{1}{L_{1}} \beta \Delta F_{\beta L_{1} ; L_{2} L_{3}}^{q} .
$$

Now we use the monotonicity of

$$
\frac{1}{|x-y|}\left(V_{q \bar{q}}^{\beta}(x-y)-V_{q \bar{q}}^{\beta}(0)\right)=-\frac{1}{|x-y|} \frac{1}{\beta} \log \frac{G(x-y)}{G(0)}
$$

(see Lemma II.5) to obtain from (II.44)

$$
\frac{1}{|x-y|} V_{q \bar{q}}^{\beta}(x-y) \leqq \lim _{L_{1} \rightarrow \infty} \frac{1}{L_{1}} \Delta F_{\beta L_{1} ; L_{2} L_{3}}^{q} .
$$

This is true for all $L_{2}, L_{3}, x, y$ with $y=x+L \hat{e}_{1}$, so in particular we can first send $L_{2}, L_{3} \rightarrow \infty$ and then $|x-y| \rightarrow \infty$.

From (II.45) one can infer an inequality between string tensions defined in different ways :

Let 't Hooft's string tension be

$$
\sigma_{{ }_{\mathrm{t}} \mathrm{H}}(\beta) \equiv \varlimsup_{L_{2}, L_{3} \rightarrow \infty} \lim _{L_{1} \rightarrow \infty} \frac{1}{L_{1}} \Delta F_{\beta L_{1} ; L_{2} L_{3}}^{q}
$$

and Polyakov's string tension

$$
\sigma_{P}(\beta) \equiv \lim _{|x-y| \rightarrow \infty} \frac{1}{|x-y|} V_{q(x) \bar{q}(y)}
$$

(where $V_{q(x) \bar{q}(y)}^{\beta}$ is to be taken in the thermodynamic limit).

Thus (II.45) implies

$$
\sigma_{{ }_{t} \mathrm{H}}(\beta) \leqq \sigma_{P}(\beta)
$$

For the sake of completeness let us note one more (trivial) inequality for string tensions at zero temperature: If $W_{L T}$ is a rectangular Wilson loop of sides $L$ and $T$, Wilson's string tension is defined as

$$
\sigma_{W} \equiv-\lim _{L, T \rightarrow \infty} \frac{1}{L T} \log \left\langle W_{L T}\right\rangle .
$$

It is then easy to see that

$$
\sigma_{P}(\infty) \leqq \sigma_{W}
$$


(this follows from the fact that $\left\langle W_{L T}\right\rangle=\left(\Psi_{L}, \mathscr{T}^{T / \tau} \Psi_{L}\right)$ with a suitable vector $\Psi_{L}$ (cf. $[44,45]$ and the fact that the trace of a positive operator dominates any diagonal matrix element). We conjecture $\sigma_{P}(\infty)=\sigma_{W}=\sigma_{{ }_{\mathrm{t}}}(\infty)$, which follows in the region of convergence of the strong coupling expansion from Münster's work [35].

The string of inequalities (II.48) and (II.50) shows that it suffices to prove confinement in the sense of 't Hooft; this is the route followed by Tomboulis [55] in his approach to the confinement problem. We also see that it suffices to prove deconfinement in the sense of Polyakov since $\sigma_{P}=0$ implies $\sigma_{\mathrm{t}_{\mathrm{H}}}=0$. To prove $\sigma_{P}=0$ at weak coupling is the subject of Sect. III.

\section{Connection to $G \times G$ Spin Systems}

Durhuus and Fröhlich in a very interesting paper [15] stressed and used the connection between $d+1$ dimensional lattice Yang-Mills theories and $d$-dimensional $G \times G$ spin models with fluctuating coupling constants. This is analogous to the well known classical relation between (static) $(d+1)$-dimensional Yang-Mills- fields and $d$ dimensional Yang-Mills-Higgs systems.

In our context this spin interpretation is both natural and very useful.

It is convenient, also in view of the later sections, to modify our notation. We label spatial points by $\mathbf{x}, \mathbf{y}$ etc. and time layers by $m, n$ etc. A spatial link is now given by $\langle\mathbf{x}, m ; \mathbf{y}, m\rangle$; we denote the corresponding gauge field by $v_{\mathbf{x y}}^{m}$. A temporal link is given by $\langle\mathbf{x}, m ; \mathbf{x}, m+1\rangle$; we denote the corresponding gauge field by $u_{\mathbf{x}}^{m}$. These temporal gauge fields will now be considered as $G$-valued spins. Their coupling in the action would be of the usual ferromagnetic nature if the spatial gauge fields $v$ were equal to the unit element; if $v_{\mathbf{x y}}^{m}$ is a pure gauge $h_{\mathbf{x}}^{m}\left(h_{\mathbf{y}}^{m}\right)^{-1}$ the coupling between the $u$ 's

$$
-S_{P}=J_{E} \operatorname{Re} \chi\left(u_{\mathbf{x}}^{m} h_{\mathbf{x}}^{m+1}\left(h_{\mathbf{y}}^{m+1}\right)^{-1}\left(u_{\mathbf{y}}^{m}\right)^{-1} h_{\mathbf{y}}^{m}\left(h_{\mathbf{x}}^{m}\right)^{-1}\right)
$$

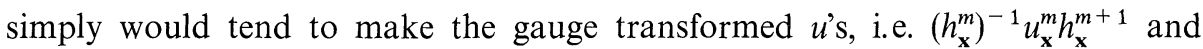
$\left(h_{\mathbf{y}}^{m+1}\right)^{-1} u_{\mathbf{y}}^{m} h_{\mathbf{y}}^{m}$ equal. In general the $v$ 's will fluctuate away from pure gauge configurations but the spatial plaquettes will still favour $v$ 's that are close to a pure gauge (i.e. will tend to suppress large magnetic fields).

It is now easy to see that we will have confinement provided the $u$-spins show exponential clustering uniformly in the random coupling field $\underset{\sim}{v}$ (see [15]):

Let

$$
\begin{gathered}
Z(\underset{v}{v})=\int \prod d u \prod_{P \text { in time dir. }} e^{-S_{P}(u, v)}, \\
\langle\cdot\rangle_{\underline{v}}=\frac{1}{Z(\underset{\sim}{v})} \int \cdot \prod d u \prod_{P \text { in time dir. }} e^{-S_{P}(u, v)} .
\end{gathered}
$$

Then

$$
\begin{aligned}
& \left\langle\overline{\chi_{q}\left(u_{L_{\mathbf{x}}}\right)} \chi_{q}\left(u_{L_{\mathbf{y}}}\right)\right\rangle \\
& =\frac{1}{Z} \int \prod d v\left\langle\overline{\chi_{q}\left(u_{L_{\mathbf{x}}}\right)} \chi_{q}\left(u_{L_{\mathbf{y}}}\right)\right\rangle_{v} Z\left(\underset{\sim}{v)} \prod_{P \text { spatial }} e^{-S_{P}(v)},\right.
\end{aligned}
$$


and therefore

$$
\left|\overline{\chi_{q}\left(u_{L \mathbf{x}}\right)} \chi_{q}\left(u_{L \mathbf{y}}\right)\right\rangle\left|\leqq \sup _{v, \alpha, \alpha^{\prime}, \beta, \beta^{\prime}}\right|\left\langle\overline{\left(u_{\mathbf{x}}\right)_{\alpha \alpha^{\prime}}}\left(u_{\mathbf{y}}\right)_{\beta \beta^{\prime}}\right\rangle \mid d_{q}^{2 L_{0}} .
$$

Durhuus and Fröhlich [15] also show how a well known expansion [5] of $\left\langle\bar{u}_{\mathbf{x}} u_{\mathbf{y}}\right\rangle_{v}$ in random paths leads to an expansion of the Wilson loop in random surfaces spanned into the loop. Here we obtain an expansion of $G(x-y)$ in random surfaces spanned between the two Polyakov loops $L_{\mathbf{x}}, L_{\mathbf{y}}$.

For completeness we give a simple derivation of this expansion in Appendix B.

We finally note that this expansion can be used to obtain very good lower bounds on the critical coupling $J_{c}$ provided $G$ is $U(1)$ or $\mathrm{SU}(2)$ (see Appendix B). Irrespective of $J_{M}$ and temperature we are sure to have confinement for $J_{E}<\tilde{J}_{c}$ where

$$
\begin{aligned}
& \tilde{J}_{c}=\frac{\varepsilon^{d-2}}{\tau} \frac{2}{\tilde{q}_{c}^{2}}=\left(d-\frac{1}{6}\right)^{-1} \text { for } \mathrm{SU}(2), \\
& \tilde{J}_{c}=\frac{\varepsilon^{d-2}}{\tau} \frac{1}{\tilde{e}_{c}^{2}}=\left(d-\frac{1}{4}\right)^{-1} \text { for } \mathrm{U}(1)
\end{aligned}
$$

These bounds are substantially better than the ones obtained by the standard strong coupling cluster expansion even in the refined form of Guerra et al. [22] [his bound for $\operatorname{SU}(2)$ is $J_{c} \geqq \frac{1}{4}(d+1)^{-1}$ ]. $d=3$

It is noteworthy that the Monte Carlo result of [27] for one time layer and

$$
J_{c}=0.31 \pm 0.04
$$

is just barely compatible with (II.55) (which gives $J_{c} \geqq 0.353$ ). If one accepts furthermore the estimated transition temperature of the $O(4)$ classical Heisenberg model $[=\mathrm{SU}(2) \times \mathrm{SU}(2)$ spin model $]$ [3]

$$
J_{c}^{O(4)}=0.47 \pm 0.01
$$

as an estimate for $J_{c}$ - which seems reasonable - the value of [27] and even the value $J_{c}=0.375 \pm$ ? given in [33] seem hard to accept. This is probably an indication that finite size effects still play an important role for lattice sizes like $8^{3}$ to $10^{3}$ which were used in those Monte Carlo computations.

This makes it more desirable to give a proof that the deconfining transition actually occurs. This is done in the following section.

\section{Breakdown of Confinement at High Temperature and Weak Coupling}

\section{One Time Layer}

The interpretation of lattice Yang-Mills theory as a $G \times G$ spin system becomes most obvious in the case of the highest possible temperature which corresponds to a lattice consisting of only one time layer. The infrared bounds of Fröhlich et al. [18] are easily transcribed to this model and yield a rather sharp lower bound for the critical coupling for the deconfining transition. 
The "Polyakov loops" consist now simply of single links in time direction biting their own tail; we label them by their spatial locations $\mathbf{x}, \mathbf{y}$ etc. and denote the corresponding gauge fields by $u_{\mathbf{x}}, u_{\mathbf{y}}$ etc. Spatial links are now simply labelled by pairs $\langle\mathbf{x y}\rangle$ and the corresponding gauge fields by $v_{\mathbf{x y}}$.

The "electric" part of the action is

$$
-S_{E}(\underline{u}, \underline{v})=J_{E} \sum_{\langle\mathbf{x y}\rangle} \operatorname{Re} \chi\left(u_{\mathbf{x}} v_{\mathbf{x y}} u_{\mathbf{y}}^{-1} v_{\mathbf{x y}}^{-1}\right),
$$

and the "magnetic" part is

$$
-S_{M}(v)=J_{M} \sum_{P} \operatorname{Re} \chi\left(v_{\partial P}\right)
$$

where the sum runs over all spatial plaquettes.

For $\chi$ we take as usual a faithful character of the compact Lie group $G$; we may then also identify the group elements with the unitary matrices of the corresponding representation. Then (III.1) can be rewritten as

$$
\begin{aligned}
-S_{E}(\underline{u}, \underline{v}) & =-\frac{1}{2} J_{E} \sum_{\langle\mathbf{x y}\rangle}\left\|\left(D_{v} u\right)_{\mathbf{x y}}\right\|^{2}+2 d\left|\Lambda_{0}\right| J_{E} \chi(\mathbb{1}) \\
& =-\frac{1}{2} J_{E} \sum_{\mathbf{x}}\left(u_{\mathbf{x}},\left(\Delta_{v} u\right)_{\mathbf{x}}\right)+2 d\left|\Lambda_{0}\right| J_{E} \chi(\mathbb{1}),
\end{aligned}
$$

where the scalar product $(\cdot, \cdot)$ is the usual Hilbert-Schmidt scalar product, i.e. $\left(u, u^{\prime}\right)=\operatorname{Tr} u^{*} u^{\prime}\left(u, u^{\prime}\right.$ are unitary matrices!), $D_{v}$ is a covariant finite difference operator:

$$
\left(D_{v} u\right)_{\mathbf{x y}}=v_{\mathbf{x y}} u_{\mathbf{y}} v_{\mathbf{x y}}^{-1}-u_{\mathbf{x}}
$$

and $\Delta_{v}=D_{v}^{*} D_{v}$ a covariant finite difference Laplacian.

After these preparations the proof of an infrared bound can to a large extent be taken over from [18], but for the benefit of the reader we give the complete argument here.

First we define a perturbed partition function $Z\left(\left\{h_{\mathbf{x}}\right\}\right)$ by replacing in III.1 or III.3 all $u_{\mathbf{x}}$ by $u_{\mathbf{x}}-h_{\mathbf{x}} \cdot \mathbb{1}\left(h_{\mathbf{x}} \in \mathbb{C}\right)$. The following bound holds:

\section{Lemma III.1.}

$$
\left|Z\left(\left\{h_{\mathbf{x}}\right\}\right)\right| \leqq Z(\{0\}) .
$$

Proof. The essential ingredient is the existence of a transfer matrix in space direction. We will first consider the 1-direction and then the other space directions. First note that (in close analogy to Lemma II.1) we have

$$
Z\left(\left\{h_{\mathbf{x}}\right\}\right)=\operatorname{Tr} P_{0} \prod_{j=1}^{L_{1}} \mathscr{T}_{j},
$$

where the operators $P_{0}, \mathscr{T}_{1}, \ldots, \mathscr{T}_{L_{1}}$ now act on the Hilbert space of squareintegrable functions of the link variables corresponding to the "transverse" directions $2,3, \ldots, d$.

The transfer matrices $\mathscr{T}_{j}$ depend on the perturbations $h_{\mathbf{x}}$ with $x_{1}=\varepsilon j$ and are again of the form

$$
\mathscr{T}_{j}=M_{j} T_{j} M_{j+1},
$$


where $M_{j}$ is multiplication by a positive function and $T_{j}$ is a convolution operator with kernel

$$
\begin{gathered}
\prod_{x_{\perp}} \exp \left(-\frac{J_{E}}{2}\left\|u_{x_{\perp}}-u_{x_{\perp}}^{\prime}-h_{\varepsilon j, x_{\perp}} \cdot \mathbb{1}\right\|^{2}\right) \\
\cdot \prod_{x_{\perp} y_{\perp}} \exp \left(-\frac{J_{M}}{2}\left\|v_{x_{\perp} y_{\perp}}-v_{x_{\perp} y_{\perp}}^{\prime}\right\|^{2}\right)
\end{gathered}
$$

where the products are over the sites $x_{\perp}$ and links $x_{\perp} y_{\perp}$, respectively, of the "transverse" lattice ( $x_{1}$ fixed) and $h_{\varepsilon j}, x_{\perp}$ is an obvious relabelling of $h_{\mathbf{x}}$.

An elementary though crucial fact is contained in

Proposition III.2. $P_{0}$ commutes with $\mathscr{T}_{j}\left(j=1, \ldots, L_{1}\right)$.

Proof. $P_{0}$ averages over ( $x_{1}$ independent) gauge transformations. So it obviously commutes with the second (magnetic) factor of III.7 as well as the corresponding magnetic part of $M$. Gauge transformations also commute with the first (electric) factor of (III.7) because

$$
\begin{aligned}
& \left\|g_{x_{\perp}} u_{x_{\perp}} g_{x_{\perp}}^{-1}-u_{x_{\perp}}^{\prime}-h \cdot \mathbb{1}\right\|^{2} \\
& \quad=\left\|u_{x_{\perp}}-g_{x_{\perp}}^{-1} u_{x_{\perp}}^{\prime} g_{x_{\perp}}-h \cdot \mathbb{1}\right\|^{2} .
\end{aligned}
$$

They also commute with the electric part of $M$ because

$$
\begin{aligned}
\| g_{x_{\perp}} u_{x_{\perp}} v_{x_{\perp} y_{\perp}} g_{y_{\perp}}^{-1}-g_{x_{\perp}} v_{x_{\perp} y_{\perp}} u_{y_{\perp}} g_{y_{\perp}}^{-1} & \quad-h_{x_{\perp}} g_{x_{\perp}} v_{x_{\perp} y_{\perp}} g_{y_{\perp}}^{-1}-h_{y_{\perp}} g_{x_{\perp}} v_{x_{\perp} y_{\perp}} g_{y_{\perp}}^{-1} \|^{2} \\
= & \left\|\left(u_{x_{\perp}}-h_{x_{\perp}} \cdot \mathbb{1}\right) v_{x_{\perp} y_{\perp}}-v_{x_{\perp} y_{\perp}}\left(u_{y_{\perp}}-h_{y_{\perp}} \cdot \mathbb{1}\right)\right\|^{2} .
\end{aligned}
$$

From this the proposition follows.

We can rewrite (III.5) as follows:

$$
\begin{aligned}
Z\left(\left\{h_{\mathbf{x}}\right\}\right) & =\operatorname{Tr} P_{0} \prod_{j=1}^{L_{1}} \mathscr{T}_{j} \\
& =\operatorname{Tr} P_{0} \prod_{j=1}^{L_{1}}\left[M_{j} T_{0}^{1 / 2}\left(T_{0}^{-1 / 2} T_{j} T_{0}^{-1 / 2}\right) T_{0}^{1 / 2} M_{j+1}\right]
\end{aligned}
$$

where $\left.T_{0} \equiv T_{j}\right|_{h_{j}=0}$.

Applying now Hölder's inequality for operators we obtain

$$
\begin{aligned}
\left|Z\left(\left\{h_{\mathbf{x}}\right\}\right)\right| & \leqq \prod_{j=1}^{L_{1}}\left\|P_{0} M_{j} T_{0}^{1 / 2}\right\|_{2 L_{1}}^{2} \prod_{j=1}^{L_{1}}\left\|T_{0}^{-1 / 2} T_{j} T_{0}^{-1 / 2}\right\|_{\infty} \\
& =\prod_{j=1}^{L_{1}}\left(\operatorname{Tr} P_{0}\left(M_{j} T_{0} M_{j}\right)^{L_{1}}\right)^{1 / L_{1}} \prod_{j=1}^{L_{1}}\left\|T_{0}^{-1 / 2} T_{j} T_{0}^{-1 / 2}\right\|_{\infty} .
\end{aligned}
$$

Here we used Proposition III.2 to move $P_{0}$ back and forth between the various factors; $\|\cdot\|_{\infty}=\|\cdot\|$ is the operator norm.

To proceed we need another fact that can essentially be transferred from [18]: 
Proposition III.3. Let $\left.T_{0} \equiv T_{j}\right|_{h_{j}=0}$. Then $\left\|T_{0}^{-1 / 2} T_{j} T_{0}^{-1 / 2}\right\| \leqq 1$.

Proof. It suffices to prove this for the simpler case where $T_{j}$ is an operator on $L^{2}(G, d g)$ with kernel $\exp \left(-\frac{J}{2}\left\|u-u^{\prime}-h_{j} \cdot \mathbb{1}\right\|^{2}\right)$ and $T_{0}$ is obtained from it by deleting $h_{j}$. Then one can use the imbedding of $G$ in a suitable $C^{n}$ to essentially diagonalize $T_{j}, T_{0}$ by Fourier transformation (the constraining $\delta$-functions do not cause any trouble). In Fourier space the shift by $h_{j}$ becomes multiplication by a phase factor so that Proposition III.3 becomes obvious. See [18] for more details.

This proposition eliminates the second factor in (III.9), i. e. it bounds $Z\left(\left\{h_{x}\right\}\right)$ in terms of partition functions where all the $h$ 's have been deleted from links in the 5 -direction. Repeating the argument with the $2, \ldots, d$-directions in place of the 1-direction completes the proof of Lemma III.1.

Lemma III.1 implies an infrared bound in the standard way: Expanding the inequality to second order in the perturbation $h$ we obtain (because the $0^{\text {th }}$ orders cancel and the first order term vanishes by translation invariance)

$$
\frac{1}{2} J_{E}^{2}\left\langle\left(\operatorname{Re}\left(\underline{u}, \Delta_{v} \underline{h}\right)\right)^{2}\right\rangle-\frac{1}{2} J_{E}\left\langle\left(\underline{h}, \Delta_{v} \underline{h}\right)\right\rangle \leqq 0 .
$$

Because $\underline{h}$ is proportional to the unit matrix the subscript $v$ on $\Delta_{v}$ can be dropped and we obtain

$$
\left\langle(\operatorname{Re}(\underline{u}, \Delta \underline{h}))^{2}\right\rangle \leqq \frac{1}{J_{E}}(\underline{h}, \Delta \underline{h}) .
$$

Replacing $\underline{h}$ by $\underline{i h}$ gives

$$
\left\langle\left(\operatorname{Im}(\underline{u}, \Delta \underline{h})^{2}\right\rangle \leqq \frac{1}{J_{E}}(\underline{h}, \underline{\Delta h}),\right.
$$

and combining (III.13) and (III.14) we get the result

$$
\left\langle|(\underline{u}, \Delta \underline{h})|^{2}\right\rangle \leqq \frac{2}{J_{E}}(\underline{h}, \Delta \underline{h}) .
$$

Remark. For $G=\mathrm{SU}(2)$ or $G=\mathrm{O}(n)$ (more generally whenever $G$ can be imbedded into a real euclidean space instead of a complex unitary space) we can obviously improve (III.15) by a factor of 2.

We write out explicitly the infrared bound we have obtained.

Theorem III.4. Let $G(\mathbf{x}-\mathbf{y}) \equiv\left\langle\overline{\operatorname{Tr} u_{\mathbf{x}}} \operatorname{Tr} u_{\mathbf{y}}\right\rangle$ and

$$
\hat{G}(\mathbf{p}) \equiv \sum_{\mathbf{x}} e^{i \mathbf{p x} / \varepsilon} G(\mathbf{x}),
$$

its Fourier transform. Then for any compact Lie group

$$
\sum_{i=1}^{d}\left(1-\cos p_{i}\right) \cdot \hat{G}(\mathbf{p}) \leqq \frac{\chi(\mathbb{1})}{J_{E}}
$$

and for $G=\mathrm{SU}(2)$ or $O(n)$

$$
\sum_{i=1}^{d}\left(1-\cos p_{i}\right) \cdot \hat{G}(\mathbf{p}) \leqq \frac{\chi(\mathbb{1})}{2 J_{E}} .
$$


Proof. This follows from (III.15) by setting $\Delta^{1 / 2} \underline{h}=\underline{g}$.

To draw any conclusions about deconfinement from the infrared bound we have to assume in addition that the expectation value of a single Polyakov loop vanishes in a periodic box. For $G=\mathrm{U}(N)$ or $\mathrm{SU}(N)$ and $\chi=\chi_{q}$ (the fundamental character) this is so because of the global invariance

$$
\left\langle\chi_{q}\left(g_{L_{\bar{x}}}\right)\right\rangle=\left\langle\chi_{q}\left(\omega g_{L \bar{x}}\right)\right\rangle
$$

where $\omega \in C(G)$ (the center of $G$ ). So we state the following result only for this physically relevant situation.

Corollary III.5. In the $\mathrm{U}(N)$ or $\mathrm{SU}(N)$ lattice Yang-Mills model with Wilson's action and maximal lattice temperature external "quarks" are liberated for

$$
\begin{gathered}
J_{E} \geqq N I(d) \quad(\text { any } N) \\
J_{E} \geqq I(d) \quad(\mathrm{U}(1) \text { or } \mathrm{SU}(2)) .
\end{gathered}
$$

In terms of the coupling constant $g^{2}=\frac{2 \varepsilon^{d-2}}{\tau J_{E}}$ this means deconfinement for

$$
\begin{gathered}
g^{2} \leqq \frac{2 \varepsilon^{d-2}}{N \tau} \frac{1}{I(d)} \quad(\text { any } N) \\
g^{2} \leqq \frac{2 \varepsilon^{d-2}}{\tau} \frac{1}{I(d)} \quad(\mathrm{U}(1) \text { or } \mathrm{SU}(2)) .
\end{gathered}
$$

(Here $I(d)$ is the integral

$$
I(d)=\frac{1}{(2 \pi)^{d}} \int\left(\sum_{i}\left(1-\cos p_{i}\right)^{-1} d^{d} p\right)
$$

which has the value

$$
0.5054620197 \text { for } d=3 \text { [18].) }
$$

Proof. The bounds (III.16) and (III.17) can be transferred to the thermodynamic limit. We need the easy bound

$$
G(0) \geqq 1,
$$

i.e.

$$
\int \hat{G}(\mathbf{p}) d^{d} p \geqq(2 \pi)^{d}
$$

[remember that $\hat{G}(\mathbf{p}) \geqq 0$ because $G$ is clearly positive definite].

Equation (III.18) follows from the Clebsch-Gordan decomposition

$$
|\operatorname{Tr} u|^{2}=1+\sum_{i} c_{i} \chi_{i}(u)
$$

where $c_{i} \geqq 0$ and $\chi_{i}$ are some irreducible characters, and the fact that by reflection positivity $\left\langle\chi_{i}(u)\right\rangle \geqq 0$.

Since $\hat{G}(\mathbf{p}) \geqq 0$ it is the density of a measure and (III.16), (III.17) say that it is absolutely continuous with respect to Lebesgue measure everywhere except 
possibly at $\mathbf{p}=0$, where it may have a discrete contribution $c \delta(p)$. In $d \geqq 3$ (III.16), (III.17) are compatible with (III.24) only if $c>0$ for sufficiently large $J_{E} . c>0$ implies long range order, that is

$$
\lim _{|\mathbf{x}| \rightarrow \infty} G(\mathbf{x})=\frac{c}{(2 \pi)^{d}} \neq 0 .
$$

According to our discussion in Sect. 2 this means absence of confinement.

Note that for $d=3$ and $G=U(1)$ or SU(2) the upper bounds (III.19), (III.20) are not too far from the lower bounds (II.55), (II.56).

$$
\begin{aligned}
& 0.353 \leqq J_{c}(\mathrm{SU}(2)) \leqq 0.505 \\
& 0.364 \leqq J_{c}(\mathrm{U}(1)) \leqq 0.505 .
\end{aligned}
$$

This concludes our discussion of the simple one layer model.

\section{Many Time Layers, Hamiltonian Limit}

Unfortunately we do not see a way to extend the simple infrared bound of the previous section to the general case. The main reason is that the basic variables we are interested in are the Polyakov loops which are now complicated nonlinear functions of the basic link variables occurring in the action.

Fröhlich et al. [18] give, however, a less elegant but more basic proof of a slightly weaker infrared bound in their appendix. It is based on the obvious fact that the transfer matrix in space direction becomes a multiple of the identity for infinite coupling between the spins. This is still true in our system and actually it is possible (with some sweat) to adapt that proof to our situation.

We again denote by $\mathscr{T}$ the transfer matrix in the 1 -direction. Furthermore we have to consider a two point function that is smeared in the transverse directions

$$
G_{h}(j) \equiv \sum_{x_{\perp} y_{\perp}} \overline{h\left(x_{\perp}\right)} G\left(\varepsilon j, x_{\perp}-y_{\perp}\right) h(y \perp), \quad(j \in \mathbb{Z})
$$

and its Fourier transform

$$
\hat{G}_{h}\left(p_{1}\right)=\sum_{j=1}^{L_{1}} e^{i p_{1} j} G_{h}(j) .
$$

The main technical result will be

Lemma III.6. There is a function $f\left(J_{E}\right)$ that falls monotonically to 0 as $J_{E} \rightarrow \infty$ such that

$$
\left(1-\cos p_{1}\right) \hat{G}_{h}\left(p_{1}\right) \leqq f\left(J_{E}\right) \sum_{x_{\perp}}\left|h\left(x_{\perp}\right)\right|^{2},
$$

or equivalently

$$
\left(1-\cos p_{1}\right) \hat{G}(\mathbf{p}) \leqq f\left(J_{E}\right)
$$

$f\left(J_{E}\right)$ may be chosen to be $\left(1+2 J_{E}^{-1} \chi(\mathbb{1})\right)^{L_{0}}-1$.

The cubic symmetry of the lattice then gives the obvious: 


\section{Corollary III.7.}

$$
\sum_{i=1}^{d}\left(1-\cos p_{i}\right) \hat{G}(\mathbf{p}) \leqq d f\left(J_{E}\right),
$$

which is the desired infrared bound implying deconfinement for $J_{E} \geqq f^{-1}\left(\frac{1}{d I(d)}\right)$.

To prove (III.29) we will make use of a lemma proven by Fröhlich et al. [18] that follows from the existence of a non-negative transfer matrix in 1-direction:

\section{Lemma III.8.}

$$
\left(1-\cos p_{1}\right) \hat{G}_{h}\left(p_{1}\right) \leqq-G_{h}(-1)+2 G_{h}(0)-G_{h}(1) .
$$

The right hand side of (III.32) is the expectation value of a double commutator:

$$
-G_{h}(-1)+2 G_{h}(0)-G_{h}(1)=\operatorname{Tr} P_{0}[\overline{L(h)},[L(h), \mathscr{T}]] \mathscr{T}^{L_{1}-1},
$$

where $\mathscr{T}$ is normalized in such a way that $\operatorname{Tr} P_{0} \mathscr{T}^{L_{1}}=1$ and

$$
L(h) \equiv \sum_{x_{\perp}} h\left(x_{\perp}\right) L_{x_{\perp}} \quad\left(L_{x_{\perp}}=\chi\left(g_{L_{x_{\perp}}}\right)\right)
$$

is a "smeared Polyakov loop." Our job will be to estimate this double commutator. It seems obvious that it will vanish as $J_{E} \rightarrow \infty$, but we will prove a more precise statement.

The transfer matrix $\mathscr{T}$ is again of the form

$$
\mathscr{T}=M \mathscr{T} M
$$

with a multiplication operator $M$ and a convolution operator $T$. $T$ is a product of a "magnetic" part involving only the $v$ variables (spatial plaquettes) and an "electric" part $T_{E}$ coupling the $u$ and $v$ variables (timelike plaquettes; see Sect. II.4). $T_{E}$ is a tensor product:

$$
T_{E}=\bigotimes_{x_{\perp}} T_{x_{\perp}}
$$

where $T_{x_{\perp}}$ has the kernel

$$
T_{x_{\perp}}\left(u_{x_{\perp}}, u_{x_{\perp}}^{\prime}\right)=\exp \left(-\frac{J_{E}}{2}\left\|u_{x_{\perp}}-u_{x_{\perp}}^{\prime}\right\|^{2}\right) .
$$

So clearly

$$
\left[T_{x_{\perp}}, \chi\left(g_{L_{y_{1}}}\right)\right]=0
$$

for $x_{\perp} \neq y_{\perp}$

Since everything else commutes the double commutator in (III.33) really only involves the double commutator

$$
\left[\overline{L(h)},\left[L(h), T_{E}\right]\right] .
$$

By (III.38) for $x_{\perp} \neq y_{\perp}$

$$
\left[\overline{L_{x_{\perp}}},\left[L_{y_{\perp}}, T_{E}\right]\right]=-\left[\overline{L_{x_{\perp}}}, T_{x_{\perp}}\right] T_{x_{\perp}}^{-1} T T_{y_{\perp}}^{-1}\left[T_{y_{\perp}}, L_{y_{\perp}}\right] .
$$


Therefore

$$
\begin{aligned}
& {\left[\overline{L(h)},\left[L(h), T_{E}\right]\right]} \\
& =-\sum_{x_{\perp}, y_{\perp}} \overline{h\left(x_{\perp}\right)} h\left(y_{\perp}\right)\left[\overline{L_{x_{\perp}}}, T_{x_{\perp}}\right] T_{x_{\perp}}^{-1} T_{E} T_{y_{\perp}}^{-1}\left[T_{y_{\perp}}, L_{y_{\perp}}\right] \\
& \quad+\sum_{x_{\perp}}\left|h\left(x_{\perp}\right)\right|^{2}\left\{T_{E} \overline{\bar{L}_{x_{\perp}}} T_{E}^{-1} L_{x_{\perp}} T_{E}-L_{x_{\perp}} T_{E} \overline{L_{x_{\perp}}}\right\}
\end{aligned}
$$

which implies

$$
\left[\overline{L(h)},\left[L(h), T_{E}\right]\right] \leqq \sum_{x_{\perp}}\left|h\left(x_{\perp}\right)\right|^{2}\left\{T_{E} \overline{L_{x_{\perp}}} T_{E}^{-1} L_{x_{\perp}} T_{E}-L_{x_{\perp}} T_{E} \overline{L_{x_{\perp}}}\right\} .
$$

[The fact that the unbounded operator $T_{E}^{-1}$ occurs requires (III.41) to be interpreted as a form inequality on a suitable dense domain.]

Now notice that $T_{E}$ is also a tensor product over time layers:

$$
T_{E}=\bigotimes_{m=1}^{L_{0}} T_{m}=\bigotimes_{x_{\perp}} \bigotimes_{m=1}^{L_{0}} T_{m x_{\perp}}
$$

If we identify $u_{x_{\perp}}^{m, m+1}$ with the unitary matrix $\left(u_{m x_{\perp}}^{a_{m} a_{m+1}}\right)$ corresponding to a representation with character $\chi$, we can write

$$
\begin{aligned}
& T_{E} \overline{L_{x_{\perp}}} T_{E}^{-1} L_{x_{\perp}} T_{E}-L_{x_{\perp}} T_{E} \overline{L_{x_{\perp}}} \\
& =\sum_{\left\{a_{m}, b_{m}\right\}}\left\{\prod_{m=1}^{L_{0}} T_{m} \overline{u_{m x_{\perp}}^{a_{m} a_{m+1}}} T_{m}^{-1} u_{m x_{\perp}}^{b_{m} b_{m+1}} T_{m}\right. \\
& \left.\quad-\prod_{m=1}^{L_{0}} u_{m x_{\perp}}^{b_{m} b_{m+1}} T_{m} \overline{u_{m x_{\perp}}^{a_{m} a_{m+1}}}\right\} .
\end{aligned}
$$

Actually we could use the factorization

$$
T_{m}=\bigotimes_{x_{\perp}} T_{m x_{\perp}}
$$

to pull out all factors $T_{m y_{\perp}}\left(y_{\perp} \neq x_{\perp}\right)$ in (III.43) because they commute with $u_{m x_{\perp}}$ (always regarded as a multiplication operator).

What remains is a sum of products of expressions of the form

$$
\overline{t u^{a a^{\prime}}} t^{-1} u^{b b^{\prime}} t \quad \text { or } \quad u^{b b^{\prime}} t \overline{u^{a a^{\prime}}}
$$

with $t$ given by its kernel

$$
t\left(u, u^{\prime}\right)=\exp \left(-\frac{J_{E}}{2}\left\|u-u^{\prime}\right\|^{2}\right) .
$$

We can shift our point of view concerning this whole computation following Eq. (III.37) by imbedding the unitary matrices in $\mathbb{C}^{N^{2}}$; this means that the Hilbert space on which our operators act will consist now of functions of $\mathbb{C}^{N^{2} \times \text { (number of links) }}$, square-integrable with respect to a $\delta$-measure that restricts $\mathbb{C}^{N^{2}}$ back to $\mathrm{U}(N)$ or $\mathrm{SU}(N)$. But we can also regard the kernels like (III.46) as kernels of operators on the $L^{2}$ with respect to Lebesgue measure. All our inequalities remain true in this interpretation, but the meaning of the symbol $T^{-1}$, for instance, 
is changed. By multiplying inequalities like (III.41) in this new interpretation from both sides with density functions that are square roots of suitable approximate $\delta$-functions we obtain - taking limits - inequalities that refer again to our original Hilbert space.

The imbedding allows us to use a Fourier transformation to compute (III.45): $t$ becomes then multiplication by $\exp \left(-\frac{1}{2 J_{E}}\|k\|^{2}\right)$ and $u^{a b}$ gets replaced by the differential operator

$$
\frac{2}{i} \frac{\partial}{\partial \bar{k}_{a b}}=\frac{1}{i}\left(\frac{\partial}{\partial \operatorname{Re} k_{a b}}+i \frac{\partial}{\partial \operatorname{Im} k_{a b}}\right) .
$$

This produces the identity

$$
\overrightarrow{t u^{a a^{\prime}}} t^{-1} u^{b b^{\prime}} t=u^{b b^{\prime}} t \overline{u^{a a^{\prime}}}+\frac{2}{J_{E}} \delta_{a b} \delta_{a^{\prime} b^{\prime}} t
$$

(III.48) can now be used to expand (III.43) in powers of $J_{E}^{-1}$. The expansion has no constant term and terminates:

$$
T_{E} \overline{L_{x_{\perp}}} T_{E}^{-1} L_{x_{\perp}} T_{E}-L_{x_{\llcorner}} T_{E} \overline{L_{x_{\perp}}}=\sum_{k=1}^{L_{0}}\left(\frac{2}{J_{E}}\right)^{k} A_{k} .
$$

$A_{k}$ can be described as follows: let

$$
A_{0} \equiv L_{x_{\perp}} T_{E} \overline{L_{x_{\perp}}}=\sum_{\left\{a_{m}, b_{m}\right\}} \prod_{m=0}^{L_{0}} u_{m x_{\perp}}^{b_{m} b_{m+1}} T_{m} \overline{u_{m x_{\perp}}^{a_{m} a_{m+1}}}
$$

Then $A_{k}$ arises from $A_{0}$ by replacing $k$ of the factors $u_{m x}^{b_{m} b_{m+1}} T_{m} \overline{u_{m x}^{a a_{m}} \overline{a_{m+1}}}$ by

$$
\frac{2}{J_{E}} \delta_{a_{m} b_{m}} \delta_{a_{m+1} b_{m+1}} T_{m}
$$

and summing over the different such possibilities $\left[\right.$ there are $\left(\begin{array}{c}L_{0} \\ k\end{array}\right)$ of them].

It is a little easier to see what this means if we reinsert this in the trace (III.33) after using the inequality

$$
[\overline{L(h)},[L(h), \mathscr{T}]] \leqq \sum_{x_{\perp}}\left|h\left(x_{\perp}\right)\right|^{2}\left\{\mathscr{T} \overline{L_{x_{\perp}}} \mathscr{T}^{-1} L_{x_{\perp}} \mathscr{T}-L_{x_{\perp}} \mathscr{T} \overline{L_{x_{\perp}}}\right\}
$$

which is a trivial consequence of (III.41). It is also convenient to rewrite the expansion (III.49) with the full transfer matrix instead of just $T_{E}$ :

$$
\mathscr{T} \overline{L_{x_{\perp}}} \mathscr{T}^{-1} L_{x_{\perp}} \mathscr{T}-L_{x_{\perp}} \mathscr{T} \overline{L_{x_{\perp}}}=\sum_{k=1}^{L_{0}}\left(\frac{2}{J_{E}}\right)^{k} B_{k} .
$$

We obtain thus from (III.33), (III.51), and (III.52)

$$
\begin{aligned}
G_{h} & (-1)-2 G_{h}(0)+G_{h}(1) \\
& \leqq \sum_{x_{\perp}} \operatorname{Tr} P_{0}\left(\mathscr{T} \overline{L_{x_{\perp}}} \mathscr{T}^{-1} L_{x_{\perp}} \mathscr{T}-L_{x_{\perp}} \mathscr{T} \overline{L_{x_{\perp}}}\right) \mathscr{T}^{L_{1}-1} \\
& =\sum_{x_{\perp}} \sum_{k=1}^{L_{0}} \operatorname{Tr}\left(P_{0} B_{k} \mathscr{T}^{L_{1}-1}\right)\left(\frac{2}{J_{E}}\right)^{k}
\end{aligned}
$$




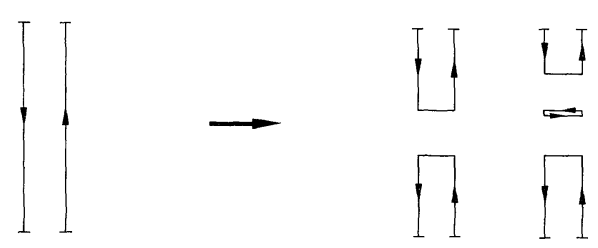

Fig. 1. Breakup of a pair of Polyakov loops into Wilson loops

where the trace now is to be understood again in the original sense, i.e. without imbedding in $\mathbb{C}^{N^{2}}$.

The terms in this last expansion can be described in a simple way: Let

$$
B_{0} \equiv L_{x_{\perp}} \mathscr{T} \overline{L_{x_{\perp}}} \text {. }
$$

Then

$$
\operatorname{Tr} P_{0} B_{0} \mathscr{T}^{L_{1}-1}
$$

is the expectation value of a pair of Polyakov loops separated by one lattice spacing in 1-direction.

$$
\operatorname{Tr} P_{0} B_{k} \mathscr{T}^{L_{1}-1}
$$

arises from (III.55) by breaking up the pair of Polyakov loops into Wilson loops by replacing in all possible ways $k$ pairs of vertical links by pairs of horizontal links (in the 1-direction). The presence of the projector $P_{0}$ in (III.56) is crucial for making this interpretation because it restores the horizontal link variables (sec Sect. II).

So we obtain the following inequality from Lemma II.6:

$$
\begin{aligned}
& \left(1-\cos p_{1}\right) \hat{G}_{h}\left(p_{1}\right) \\
& \quad \leqq \sum_{x_{\perp}}\left|h\left(x_{\perp}\right)\right|^{2} \sum_{k=1}^{L_{0}}\left(\frac{2}{J_{E}}\right)^{k} \sum_{C_{1}, \ldots, C_{k}}\left\langle W\left(C_{1}\right) \ldots W\left(C_{k}\right)\right\rangle .
\end{aligned}
$$

Here the last sum is over all $\left(\begin{array}{c}L_{0} \\ k\end{array}\right)$ sets of Wilson loops $\left\{C_{1} \ldots C_{k}\right\}$ that arise from a pair of Polyakov loops separated by one lattice unit in the 1-direction by applying the breakup procedure described above (see Fig. 1). Because of translation invariance we can ignore the positions $x_{\perp}$ of those loops.

If we now apply the trivial bound $|W(C)| \leqq \chi(\mathbb{1})$, we obtain from (III.57)

$$
\begin{aligned}
(1 & \left.-\cos p_{1}\right) \hat{G}_{h}\left(p_{1}\right) \\
& \leqq \sum_{x_{\perp}}\left|h\left(x_{\perp}\right)\right|^{2} \sum_{k=1}^{L_{0}}\left(\frac{2}{J_{E}}\right)^{k} \sum_{C_{1}, \ldots, C_{k}} \chi(\mathbb{1})^{k} \\
& =\sum_{x_{\perp}}\left|h\left(x_{\perp}\right)\right|^{2} \sum_{k=1}^{L_{0}}\left(\frac{2}{J_{E}}\right)^{k} \chi(\mathbb{1})^{k}\left(\begin{array}{c}
L_{0} \\
k
\end{array}\right) \\
& =\sum_{x_{\perp}}\left|h\left(x_{\perp}\right)\right|^{2}\left[\left(1+\frac{2 \chi(\mathbb{1})}{J_{E}}\right)^{L_{0}}-1\right] .
\end{aligned}
$$


So we have proven Lemma III.6 in the form

$$
\left(1-\cos p_{1}\right) \hat{G}(\mathbf{p}) \leqq\left[\left(1+\frac{2 \chi(\mathbb{1})}{J_{E}}\right)^{L_{0}}-1\right],
$$

and Corollary III.7 in the form

$$
\sum_{i}\left(1-\cos p_{i}\right) \hat{G}(\mathbf{p}) \leqq d\left[\left(1+\frac{2 \chi(\mathbb{1})}{J_{E}}\right)^{L_{0}}-1\right] .
$$

If we specialize to $L_{0}=1$ we realize that we have lost a factor $2 d$ in comparison with (III.16). This same factor also occurred in [18] and is typical for this "double commutator proof." Presumably an inequality

$$
\sum_{i}\left(1-\cos p_{i}\right) \hat{G}(\mathbf{p}) \leqq \frac{1}{2}\left[\left(1+\frac{2 \chi(\mathbb{1})}{J_{E}}\right)^{L_{0}}-1\right]
$$

is true for all $L_{0}$ but we have no proof for it. Certain improvements are again possible if there are reality properties:

If the representation to which $\chi$ belongs is real orthogonal, we may use an imbedding of our variables in $\mathbb{R}^{N^{2}}$ instead of $\mathbb{C}^{N^{2}}(N=\chi(\mathbb{1}))$. This will lead to the replacement of $J_{E}$ by $2 J_{E}$ in (III.48) and all bounds. Again this remark is somewhat academic because it does not apply in the cases of physical interest $[G=\mathrm{U}(N)$ or $\mathrm{SU}(N), \chi$ the fundamental character].

Another improvement is possible for $G=\mathrm{SU}(2)$ (or more generally whenever $\chi$ is real): We split the $u$-variables in (III.43) etc. in their real and imaginary parts. This splits each term in the sum into $2^{2 L_{0}}$ new terms, but due to the reality of the trace only the terms contribute in which for each $\prod_{m}\left(\operatorname{Re} u_{m}+i \operatorname{Im} u_{m}\right)$ we pick the imaginary part an even number of times. Compared with the general case in which only the whole expression, not the individual loops are real, we gain a factor of two in the infrared bound (III.59) (see [2]).

Let us write out explicitly what we can learn about deconfinement from Lemma III.6 and these remarks:

Theorem III.7. In the $\mathrm{U}(N)$ or $\mathrm{SU}(N)$ lattice Yang-Mills theory with Wilson's action and temperature $T$ (i.e. $L_{0}=(\tau T)^{-1}$ time layers) external "quarks" are liberated if

$$
\begin{gathered}
J_{E} \geqq 2 N\left[\left(1+\frac{1}{d I(d)}\right)^{\tau T}-1\right]^{-1} \quad(\text { any } N), \\
J_{E} \geqq 4\left[\left(1+\frac{2}{d I(d)}\right)^{\tau T}-1\right]^{-1} \quad(G=\mathrm{SU}(2)) .
\end{gathered}
$$

In terms of the coupling constant this means deconfinement for

$$
\begin{array}{ll}
g^{2} \leqq \frac{\varepsilon^{d-2}}{\tau N}\left[\left(1+\frac{1}{d I(d)}\right)^{\tau T}-1\right] & (\text { any } N), \\
g^{2} \leqq \frac{\varepsilon^{d-2}}{2 \tau}\left[\left(1+\frac{2}{d I(d)}\right)^{\tau T}-1\right] & (G=\mathrm{SU}(2)) .
\end{array}
$$


Proof. This is essentially the same as the proof of Corollary III.5.

It is a gratifying fact that the bounds stay nontrivial in the Hamiltonian limit $\tau \rightarrow 0$. We have

Corollary III.8. In the Hamiltonian lattice gauge model of Kogut and Susskind (i.e. the limit $\tau \rightarrow 0$ of Wilson's model) with gauge group $\mathrm{U}(N)$ or $\mathrm{SU}(N)$ quarks are liberated for

$$
\begin{array}{ll}
g^{2} \leqq \frac{\varepsilon^{d-2} T}{N} \log \left(1+\frac{1}{d I(d)}\right) & (\text { any } N) \\
g^{2} \leqq \frac{\varepsilon^{d-2} T}{2} \log \left(1+\frac{2}{d I(d)}\right) & (G=\mathrm{SU}(2))
\end{array}
$$

Proof. This is trivial if one notices that the infrared bound (III.59) has a limit as $\tau \rightarrow 0$.

We can also say something about 't Hooft's $N \rightarrow \infty$ limit with $\bar{g}^{2}=g^{2} N$ fixed :

Corollary III.9. In 't Hooft's $N \rightarrow \infty$ limit quarks are liberated for

$$
\bar{g}^{2} \leqq \frac{\varepsilon^{d-2}}{\tau}\left[\left(1+\frac{1}{d I(d)}\right)^{\tau T}-1\right] .
$$

Proof. Obvious.

This concludes our discussion of the general case. In the next section we will discuss abelian models in some more detail.

\section{Some Further Results for Abelian Models}

For abelian models there is a way of proving the infrared bounds (actually a slightly stronger version of it) in a more "natural" way that does not rely on the imbedding of the group in a matrix space.

An additional advantage of this procedure is that it allows more general forms of the action, such as the so-called Villain action which is considered frequently $[13,57]$.

We consider gauge groups $U(1)$ (or $Z_{N}$; direct products of such groups can be handled just as well but they would pollute our notation too much) and actions characterized by an electric plaquette coupling

$$
e^{-S_{P}\left(g_{\hat{o} P}\right)}=\sum_{\tau \in \hat{G}} m_{\tau}\left(J_{E}\right) \chi_{\tau}\left(g_{\partial P}\right) .
$$

Here

$$
g_{\partial P}=u_{\mathbf{x}}^{m} v_{\mathbf{x y}}^{m+1}\left(u_{\mathbf{y}}^{m}\right)^{-1} v_{\mathbf{y x}}^{m}
$$

for a plaquette $p$ with corners $(\mathbf{x}, m),(\mathbf{x}, m+1),(\mathbf{y}, m+1),(\mathbf{y}, m)$. The sum in (III.69) is over the irreducible characters $\tau$ of $G$, the coefficients $m_{\tau}$ and $e^{-S_{p}}$ are assumed to be $\geqq 0$ and we assume

$$
\lim _{J_{E} \rightarrow \infty} m_{\tau}\left(J_{E}\right)=1 .
$$


The magnetic coupling will be of the same form as (III.69), but the coefficients $m_{\tau}$ may be different from the ones in (III.69). We only need that they (as well as $e^{-S_{p}}$ ) are nonnegative to assure the existence of a nonnegative transfer matrix in space direction.

Typical examples for (III.69) are the Villain action for $G=\mathrm{U}(1)$

$$
m_{\tau}\left(J_{E}\right)=\exp \left(-\frac{1}{J_{E}} \tau^{2}\right)
$$

(here $\tau$ is an integer) and Wilson's action which for $G=\mathrm{U}(1)$ is characterized by

$$
m_{\tau}\left(J_{E}\right)=I_{\tau}\left(J_{E}\right)
$$

$\left(I_{\tau}\right.$ is the modified Bessel function of order $\tau$ ).

We proceed as in the previous section up to Eq. (III.41) which said

$$
\begin{aligned}
& {\left[\overline{L(h)},\left[L(h), T_{E}\right]\right]} \\
& \quad \leqq \sum_{x_{\perp}}\left|h\left(x_{\perp}\right)\right|^{2}\left\{T_{E} \overline{L_{x_{\perp}}} T_{E}^{-1} L_{x_{\perp}} T_{E}-L_{x_{\perp}} T_{E} \overline{L_{x_{\perp}}}\right\}
\end{aligned}
$$

The right hand side can of course again be factored over time layers:

$$
\begin{aligned}
& T_{E} \overline{L_{x_{\perp}}} T_{E}^{-1} L_{x_{\perp}} T_{E}-L_{x_{\perp}} T_{E} \overline{L_{x_{\perp}}} \\
& \quad=\prod_{m=1}^{L_{0}} T_{m} \bar{u}_{m x_{\perp}} T_{m}^{-1} u_{m x_{\perp}} T_{m}-\prod_{m=1}^{L_{0}} u_{m x_{\perp}} T_{m} \bar{u}_{m x_{\perp}} .
\end{aligned}
$$

The point is now that the right hand side of (III.75) is again a convolution operator; this can be seen by using Fourier transformation which diagonalizes everything. Again [as in (III.45)] we have to deal with sums of products of expressions of the form

$$
t \bar{u} t^{-1} u t \text { or } u t \bar{u}
$$

where $u$ is multiplication by $e^{i \varphi}$ and $t$ is convolution by

$$
t(\varphi)=\sum_{\tau} m_{\tau} e^{i \tau \varphi}
$$

Therefore $t \bar{u} t^{-1} u t$ is convolution by

$$
\sum_{\tau} \frac{m_{\tau}^{2}}{m_{\tau+1}} e^{i \tau \varphi}
$$

and $u t \bar{u}$ is convolution by

$$
\sum_{\tau} m_{\tau-1} e^{i \tau \varphi}
$$

Therefore

$$
t \bar{u} t u t=u t \bar{u}+r
$$

where $r$ is convolution by

$$
r(\varphi)=\sum_{\tau} m_{\tau-1}\left(\frac{m_{\tau}^{2}}{m_{\tau+1} m_{\tau-1}}-1\right) .
$$


So clearly $r$ obeys the form inequality

$$
r \leqq g\left(J_{E}\right) u t \bar{u}
$$

with

$$
g\left(J_{E}\right)=\sup _{\tau}\left(\frac{m_{\tau}^{2}}{m_{\tau+1} m_{\tau-1}}-1\right) .
$$

We can now expand (III.75) in powers of $r$. Using the inequality (III.81) in each term we can resum to obtain

$$
\begin{aligned}
& T_{E} \overline{L_{x_{\perp}}} T_{E}^{-1} L_{x_{\perp}} T_{E}-L_{x_{\perp}} T_{E} \overline{L_{x_{\perp}}} \\
& \quad \leqq\left[\left(1+g\left(J_{E}\right)\right)^{L_{0}}-1\right] L_{x_{\perp}} T_{E} \overline{L_{x_{\perp}}},
\end{aligned}
$$

and

$$
\begin{aligned}
{\left[\overline{L(h)},\left[L(h), T_{E}\right]\right] \leqq } & \sum_{x_{\perp}}\left|h\left(x_{\perp}\right)\right|^{2} L(h) T_{E} \overline{L(h)} \\
& \cdot\left[\left(1+g\left(J_{E}\right)\right)^{L_{0}}-1\right] .
\end{aligned}
$$

As in the previous section this yields an infrared bound:

Lemma III.9. For an abelian model characterized by the electric coupling function (III.69) an infrared bound of the following form holds:

$$
\sum_{i=1}^{d}\left(1-\cos p_{i}\right) \hat{G}(\mathbf{p}) \leqq d f\left(J_{E}\right) \equiv d\left[\left(1+g\left(J_{E}\right)\right)^{L_{0}}-1\right],
$$

where

$$
g\left(J_{E}\right)=\sup _{\tau}\left(\frac{m_{\tau}^{2}}{m_{\tau+1} m_{\tau-1}}-1\right) .
$$

Of course this result is useful only if $g\left(J_{E}\right) \rightarrow 0$ as $J_{E} \rightarrow \infty$. We check this for the Villain action. There

$$
g\left(J_{E}\right)=\exp \frac{1}{J_{E}}-1
$$

and hence

$$
f\left(J_{E}\right)=\exp \frac{L_{0}}{J_{E}}-1 .
$$

This clearly implies deconfinement for

$$
J \geqq f^{-1}\left(\frac{1}{d I(d)}\right)=\left[\tau T \log \left(1+\frac{1}{d I(d)}\right)\right]^{-1} .
$$

For the Wilson action

$$
g\left(J_{E}\right)=\left(\frac{I_{0}\left(J_{E}\right)}{I_{1}\left(J_{E}\right)}\right)^{2}-1
$$


and hence

$$
\begin{aligned}
f\left(J_{E}\right) & =\left(\frac{I_{0}\left(J_{E}\right)}{I_{1}\left(J_{E}\right)}\right)^{2 L_{0}}-1 \\
& =\frac{L_{0}}{J_{E}}+O\left(J_{E}^{-2}\right) .
\end{aligned}
$$

The proof of (III.88) requires some gymnastics with Bessel functions and is given in Appendix C. (III.89) is slightly stronger than (III.60), at least for large $J_{E}$.

We refrain from giving the resulting bound for the deconfining transition because it would look somewhat messy.

\section{Concluding Remarks}

Let us try to put into perspective what has been proven and point out some important open questions.

We have shown that lattice Yang-Mills theory with Wilson's action in space dimension three (or more) will possess, as soon as a non-zero temperature is turned on, a weak coupling phase without confinement in addition to the well known confining strong coupling phase.

The situation is slightly different for the Kogut-Susskind Hamiltonian model: There we have shown that confinement will disappear for any coupling provided the temperature is high enough [cf. (III.66) and (III.67)]. Convergence of the strong coupling expansion sketched by Kogut and Susskind [26] has not been established rigorously; we have no doubt that one could do this, however, and provide a proof that the string tension $\sigma_{p}$ does not vanish for $g^{2}>\bar{g}^{2}(T)$ (obviously $\bar{g}^{2}$ cannot be independent of $T$ here).

It is expected that the unconfining phase behaves like a "gluon plasma" and shows Debye screening (i.e. exponential clustering) at high enough temperature; the technology to establish this has been provided by Brydges and Federbush [6], but some work is needed to adapt their methods to this problem; the nonlocal effective coupling between the Polyakov loops will not make this task easier.

In [3] we pointed out that there is even the possibility of two phase transitions: First from the confining to a Coulombic phase and then to a Debye screened plasma. For $\mathrm{SU}(N)$ with $N$ large this would not seem implausible because of the analogy with $Z_{N}$ models [53]. But most physicists seem to regard it as unlikely for $N=2,3$. In fact Svetitsky and Yaffe [53] argue by analogy with the Ising or Potts models, respectively, that for $N=2$ there should be one second order and for $N=3$ one first order deconfining transition.

Our inequality (II.48) shows that at positive temperature and small enough coupling also 't Hooft's string tension vanishes; this might have been difficult to show directly.

Our proof in Sect. III. 2 made use of the explicit form of Wilson's action, at least for the "electric" coupling. So one might wonder how model dependent the results are. But one should note that first of all the form of the magnetic coupling was fairly arbitrary; it only had to be compatible with reflection positivity, i.e. a positive transfer matrix in space direction. 
Secondly, the $\tau$-continuum (=Hamiltonian) limit will be largely independent of the precise form of the electric coupling; for instance Theorem II. 2 shows that the heat kernel ( =Villain) action $[13,57]$ will produce the same "electric" part in the $\tau$-continuum limit as Wilson's. But since we have an infrared bound for $\tau=0$, a similar bound must hold already for $\tau>0, \tau$ small enough. This shows that deconfinement occurs for a wide class of lattice models.

To see the strengths and weaknesses of our bounds it is instructive to discuss the limiting cases $J_{M} \rightarrow 0$ and $J_{M} \rightarrow \infty$. The peculiar feature of our method is that it is completely independent of $J_{M}$.

For $J_{M}=0$ we obtain the "ultralocal" model already discussed in [38]; for $\tau=0$ this is the strong coupling limit of the Kogut-Susskind model [26] around which a strong coupling expansion has to be constructed. The ultralocal model shows confinement at all couplings (and in any dimension!) at zero temperature. For finite temperature it becomes structurally identical to a one time layer model; this is easiest to see for the heat kernel (Villain) action characterized by a transfer matrix (in time direction) that is simply given by the heat kernel:

$$
\mathscr{T}\left(\underset{\sim}{v}, v_{\sim}^{\prime}\right)=\prod_{\langle\mathbf{x y}\rangle} e^{\frac{g^{2}}{2} \Delta}\left(v_{\mathbf{x y}} v_{\mathbf{x y}}^{\prime-1}\right)
$$

( $\Delta$ is the Laplace-Beltrami operator on the group $G$ ). So the ultralocal Villain model will depend only on the product

$$
L_{0} g^{2}=\frac{g^{2}}{\tau T}
$$

and we may put $L_{0}=1$ and change $g^{2}$ accordingly.

For more general ultralocal actions we still have the structure of the one layer model, but with a more complicated electric coupling arising from the $L_{0}$-fold convolution of $\mathscr{T}$ with itself.

In any case these models can all be regarded as $G \times G$ spin models with some random coupling provided by the $v$ variables (cf. Sect. III.1). The random couplings might be expected to create disorder, thereby making deconfinement harder. For abelian models this follows from Ginibre's inequalities [20]. But for nonabelian groups the random couplings reduce the symmetry from $G$ to $C(G)$ and might actually thereby facilitate ordering. This certainly would be relevant for $d=2^{1}$. We think that for $d \geqq 3$ the effect of the random couplings is not so dramatic. Since the infrared bound gives excellent results for spin models without random couplings we conjecture:

The infrared bound gives a bound on the transition point $\bar{g}^{2}$ that is close $(\sim 10 \%)$ to the actual value for the ultralocal $\left(J_{M}=0\right)$ models, if we either apply it to the effective one layer version of that model or "steal back" the factor $2 d$ lost in the double commutator proof.

The other extreme case $J_{M} \rightarrow \infty$ is also easy to understand. In this limit the "magnetic fields" $v_{\partial P}$ are frozen out, the $v$ variables become a pure gauge and can be gauged away. One is left with a stack of $L_{0}$ copies of the $G \times G$ spin model; the

1 We thank G. Mack for reminding us of this fact 
transition point $\bar{g}^{2}$ becomes therefore independent of $L_{0}$ and will be close to the infrared bound result for one layer:

The infrared bound for one layer gives a bound on the transition point $\bar{g}^{2}$ that is close $(\sim 10 \%)$ to the actual value for the $J_{M} \rightarrow \infty$ limit of lattice Yang-Mills models.

It is now highly plausible that the true transition point will lie between the values obtained for $J_{M}=0$ and $J_{M} \rightarrow \infty$. For abelian models this is of course again implied by Ginibre's inequalities.

The conventional renormalization group philosophy predicts that all physical quantities with the dimension of a mass should behave like

$$
\left(g^{2}\right)^{-\beta_{1} / 2 \beta_{0}} \exp \left(-\frac{1}{2 g^{2} \beta_{0}}\right),
$$

so in particular $T_{c}$, the critical temperature for deconfinement should go like

$$
T_{c} \sim\left(g^{2}\right)^{-\frac{\beta_{1}}{2 \beta_{0}}} \exp \left(-\frac{1}{2 g^{2} \beta_{0}}\right) .
$$

It is not surprising that our bounds completely fail to produce such a behavior. To actually prove that a physical quantity shows the correct scaling behavior would almost be equivalent to the construction of the continuum limit and is clearly beyond the scope of this paper. If, on the other hand, we assume the correct scaling behavior (the way it is normally done in the physics literature) and make the somewhat bold assumption that even for only one layer we can be close to the scaling region, it is possible to extract a physical value of the transition temperature that is not unreasonable (a few hundred $\mathrm{MeV}$ ); this is done by simply pretending that the transition temperature expressed in units of the string tension (at the same coupling but zero temperature) does not change much when we go to the continuum limit.

We should maybe mention that it is not an inherent weakness of the infrared bound technique but rather our inability to control the continuum limit that prevents us from establishing deconfinement in the continuum. It should be remembered that infrared bounds were first found and applied in a continuum model [the $\left(\phi^{2}\right)^{2}$ model in $d=3$ ].

There is one more important open question: What becomes of the deconfining transition when dynamical quarks (or maybe Higgs fields in the fundamental representation) are included? Obviously the Polyakov loops will lose their diagnostic value because, physically speaking, external quarks are shielded by dynamical ones; mathematically speaking we lose the global symmetry that assured $\left\langle\chi\left(g_{2}\right)\right\rangle=0$ in the pure Yang-Mills model. Since a fundamental Higgs field (and probably dynamical quarks as well) has an effect on the system that is very close to the effect of a magnetic field on a ferromagnet [46] one might expect that there is no longer a transition. Of course we still expect qualitatively different behavior at high and low temperatures but maybe not a sudden (nonanalytic) change.

If our matter fields are Higgs fields in the adjoint representation (such as in most grand unified theories) the Polyakov loops can still be used to test for confinement and our methods can be extended to show the existence of a 
deconfining transition. Much less clear is the existence of the supposed first order transition between a "Higgs phase" at low and a "symmetric phase" at high temperature $[12,28]$ that has led to so much cosmological speculation $[23,51]$.

All this shows in our opinion that it is worth investigating the phase structure of gauge theories at finite temperature in more detail and that it is also essential to sharpen the diagnostic tools that should distinguish between the various phases. This will hopefully lead to a better understanding of what is meant by "confinement" in the full theory and what such terms as "Higgs phase" or phase with "unbroken gauge symmetry" really mean. The (dis)order parameters proposed by Mack and Meyer [30], Bricmont and Fröhlich [4] as well as Fredenhagen [17] might be useful steps in that direction.

To really understand what deconfinement means for the full theory would, however, require the development of a precise (quasi-)particle concept for field theories at finite temperature and the existence of tools to analyze concrete models from this point of view.

\section{Appendix A. The $\tau$-Continuum Limit}

In this appendix we give the proof of Theorem II.2. For notational convenience we set $\varepsilon=1$ and $g^{2}=1$ (it will become clear that this is not a significant loss of generality). We also choose a basis $\left\{i L_{a}\right\}$ of the Lie algebra $\mathfrak{g}$ of our gauge group $G$ with the orthonormality property

$$
\chi\left(L_{a} L_{b}\right) \equiv \operatorname{Tr} L_{a} L_{b}=\delta_{a b},
$$

where $\chi$ is the character used in the action and we denote by $L_{a}$ also the representatives of the generators in the faithful representation belonging to $\chi . L_{a}^{(q)}$ will denote the representative of the generator $L_{a}$ in the representation labelled by $q$.

We first consider the easier case of the heat kernel (Villain) action and the corresponding transfer matrix $\mathscr{T}=\mathscr{T}_{H}$. We have to show that

$$
\mathscr{T}(\tau)^{\beta / \tau}=\left(e^{-\frac{\tau}{2} V} e^{\frac{\tau}{2} \Delta} e^{-\frac{\tau}{2} V}\right)^{\beta / \tau}
$$

goes to $\exp \beta\left(\frac{1}{2} \Delta-V\right)$ as $\tau \rightarrow 0$ in all $J_{p}$ norms ( $p \geqq 1$ ). Without loss of generality we will consider only $p=1$.

Strong convergence follows from the Trotter product formula [43] if $\tau \rightarrow 0$ through a sequence such that $\beta / \tau$ is an integer. For the general case we write $\beta=n \tau+\varepsilon$ with $0 \leqq \varepsilon<\tau, n \in \mathbb{N}$. Then

$$
\mathscr{T}(\tau)^{\beta / \tau}=\mathscr{T}(\tau)^{n} \mathscr{T}(\tau)^{\varepsilon / \tau},
$$

and strong convergence follows by a simple $3 \varepsilon$ argument:

$$
\begin{aligned}
\left(\mathscr{T}(\tau)^{\beta / \tau}-e^{\beta\left(\frac{1}{2} \Delta-V\right)}\right) \Psi= & \mathscr{T}(\tau)^{n}\left(\mathscr{T}(\tau)^{\varepsilon / \tau}-\mathbb{1}\right) \Psi \\
& +\left(\mathscr{T}(\tau)^{n}-e^{n \tau\left(\frac{1}{2} \Delta-V\right)}\right) \Psi \\
& +e^{n \tau\left(\frac{1}{2} \Delta-V\right)}\left(\mathbb{1}-e^{\varepsilon\left(\frac{1}{2} \Delta-V\right)}\right) \Psi
\end{aligned}
$$


Each term in (A.3) goes to zero: Note that $\mathscr{T}(\tau)^{\varepsilon / \tau} \rightarrow \mathbb{1}$ strongly because $\mathscr{T}(\tau) \rightarrow \mathbb{1}$ strongly.

To obtain $J_{1}$ or more generally $J_{p}$ convergence we use Grümm's theorem [21] which says that strong convergence together with convergence of the $J_{p}$ norms of the approximants to the $J_{p}$ norm of the limiting operator implies $J_{p}$ convergence (see [49]) for a sharper version).

So it remains to prove

Lemma A.1. Let $G$ be a compact Lie group and $V$ be uniformly Lipschitz continuous on $G$. Then

$$
\begin{aligned}
F(\tau) & \equiv \operatorname{Tr} \mathscr{T}(\tau)^{\beta / \tau} \\
& =\operatorname{Tr}\left(e^{-\frac{\tau}{2} V} e^{\tau-2 \Delta} e^{-\frac{\tau}{2} \Delta}\right)^{\beta / \tau}
\end{aligned}
$$

converges towards

$$
\operatorname{Tr} e^{\beta\left(\frac{1}{2} \Delta-V\right)} \quad \text { as } \quad \tau \rightarrow 0 \text {. }
$$

Proof. A proof can be based on the Feynman-Kac formula for Brownian motion on $G$ (see [31]).

For completeness we give an independent proof that does not use the Feynman-Kac formula (but is inspired by it). Expanding (A.4) in powers of $V$ we obtain (with $N=\beta / \tau$ )

$$
F(\tau)=\sum_{k \geqq 0} \frac{1}{k !} \sum_{\Sigma k_{i}=k} \frac{k !}{k_{1} ! \ldots k_{N} !} \operatorname{Tr} \prod_{i=1}^{N}\left((-\tau V)^{k_{1}} e^{\frac{1}{2} \tau \Delta}\right) ;
$$

$\operatorname{Tr} \exp \beta\left(\frac{1}{2} \Delta-V\right)$ can be similarly expanded (the so-called Duhamel-Phillips expansion $[11,47])$.

$$
\begin{aligned}
\operatorname{Tr} e^{\beta\left(\frac{1}{2} \Delta-V\right)}= & \sum_{k \geqq 0} \int_{0 \leqq t_{1} \leqq \ldots \leqq t_{k} \leqq \beta} d t_{1} \ldots d t_{k} \\
& \cdot \operatorname{Tr} \prod_{i=1}^{k}\left(e^{\frac{1}{2} t_{i} \Delta}(-V) e^{-\frac{1}{2} t_{i} \Delta}\right) e^{\frac{1}{2} \beta \Delta} .
\end{aligned}
$$

It is seen easily that the $k^{\text {th }}$ term of both (A.5) and (A.6) is bounded by

$$
\frac{\beta^{k}\|V\|^{k}}{k !} \operatorname{Tr} e^{\frac{1}{2} \beta \Delta}
$$

so that both series converge absolutely and (A.5) converges uniformly in $\tau$. So it suffices to show convergence term by term. We rewrite the $k^{\text {th }}$ term of (A.5) as

$$
\begin{aligned}
& \frac{1}{k !} \sum_{l_{1} \ldots l_{k}=0}^{N-1} \tau^{k} \operatorname{Tr}\left(T \prod_{i=1}^{k}\left(-V\left(\tau l_{i}\right)\right)\right) e^{\frac{\beta}{2} \Delta} \\
& \quad=\frac{1}{k !} \int_{0}^{\beta} d t_{1} \ldots d t_{k} \operatorname{Tr}\left(T \prod_{i=1}^{k}\left(-V\left(\tau\left[\frac{t_{i}}{\tau}\right]\right)\right)\right) e^{\frac{\beta}{2} \Delta},
\end{aligned}
$$


where we used the notation

$$
V(t) \equiv e^{\frac{t}{2} \Delta} V e^{-\frac{t}{2} \Delta}
$$

and $[x]$ denotes the integer part of $x . T$ is an ordering symbol saying that the factors should be written with the "times" $t_{i}$ increasing from left to right. Similarly the $k^{\text {th. }}$ term of (A.6) can be written as

$$
\frac{1}{k !} \int_{0}^{\beta} d t_{1} \ldots d t_{k} \operatorname{Tr} T \prod_{i=1}^{k}\left(-V\left(t_{i}\right)\right) e^{\frac{\beta}{2} \Delta} .
$$

Equation (A.7) can be read as a Riemann sum approximation of (A.8). Since the integrands of both (A.7) and (A.8) are bounded by

$$
\|V\|_{\infty}^{k} \operatorname{Tr} e^{\frac{\beta}{2} \Delta}
$$

it suffices to show convergence of the integrands (by the dominated convergence theorem).

So we have to estimate an expression of the form

$$
\operatorname{Tr}\left[T \prod_{i=1}^{k}\left(-V\left(t_{i}\right)\right)-T \prod_{i=1}^{k}\left(-V\left(s_{i}\right)\right)\right] e^{\frac{\beta}{2} \Delta},
$$

where $\left|t_{i}-s_{i}\right|<\tau(i=1, \ldots, k)$ and $0 \leqq t_{1}, \ldots, t_{k}, s_{1}, \ldots, s_{k} \leqq \beta$. Note that (A.9) depends only on the differences $t_{2}-t_{1}, \ldots, t_{k}-t_{k-1}, t_{1}-t_{k}+\beta$ by the cyclicity of the trace. Telescoping (A.9) and using cyclicity of the trace in a similar way we obtain $k$ terms of the form

$$
R_{\tau}(t, s) \equiv \operatorname{Tr}(V(t)-V(s)) \prod_{i=2}^{k} V\left(u_{i}\right) e^{\frac{\beta}{2} \Delta},
$$

where $|t-s|<\tau ; 0 \leqq t, s \leqq u_{2} \leqq \ldots \leqq u_{k} \leqq \beta$.

Rewriting (A.10) in terms of integral kernels and using the positivity of the heat kernel we obtain the bound

$$
\begin{aligned}
\left|R_{\tau}(t, s)\right| \leqq & \|V\|_{\infty}^{k-1} \int d \underset{\sim}{g} \underset{\sim}{h}|V(\underset{\sim}{g})-V(\underset{\sim}{h})| \\
& \cdot e^{\frac{1}{2}|t-s| \Delta}(\underset{\sim}{g} \underset{\sim}{\operatorname{h}})^{\frac{1}{2}(\beta-|t-s|) \Delta}(\underset{\sim}{h}, \underset{\sim}{g}) .
\end{aligned}
$$

Using the uniform Lipschitz property of $V$ this implies

$$
\begin{aligned}
\left|R_{\tau}(t, s)\right| & \leqq C\|V\|_{\infty}^{k-1} \int d \underset{\sim}{g} \underset{\sim}{\operatorname{d} d} \underset{\sim}{g} \underset{\sim}{\operatorname{h}} \underset{\sim}{e^{\frac{1}{2}|t-s| \Delta}}(\underset{\sim}{g}, \underset{\sim}{h}) \\
& =C\|V\|_{\infty}^{k-1} \int d \underset{\sim}{g} d(\underset{\sim}{g}, \mathbb{1}) e^{\frac{1}{2}|t-s| \Delta}(\underset{\sim}{g}, \mathbb{1}),
\end{aligned}
$$

where $d(g, \underset{\sim}{h})$ is the geodesic distance between $g$ and $\underset{\sim}{h}$ and we estimated the second heat kernel in (A.11) by a constant.

Now we need the well known fact that the heat kernel $e^{\frac{1}{2} t \Delta}(t, h)$ for small $t$ behaves like const $t^{-D / 2} \exp \left(-\frac{1}{2 t} d(\underset{\sim}{g} \underset{\sim}{h})^{2}\right)$ or, more precisely, that

$$
e^{\frac{1}{2} t \Delta}(\underset{\sim}{g} \underset{\sim}{h}) \leqq \operatorname{const} t^{-D / 2} e^{-\frac{\alpha}{2 t} d(g, h)^{2}}
$$


where $D$ is the dimension of the manifold $G^{\text {number of linksin } \Lambda_{0}}$ and $\alpha<1$ is some constant (see for instance $[32,42]$ ). Inserting (A.13) in (A.12) shows

$$
\begin{aligned}
\left|R_{\tau}(s, t)\right| & \leqq \text { const } \sqrt{|t-s|} \\
& \leqq \text { const } \sqrt{\tau} .
\end{aligned}
$$

The proof of Lemma A.1 is now complete.

To prove the analog of Lemma A.1 for the Wilson transfer matrix $\mathscr{T}_{W}$ it suffices (again by Grümm's theorem) to show that

$$
\operatorname{Tr} \mathscr{T}_{W}(\tau)^{\beta / \tau}-\operatorname{Tr} \mathscr{T}_{H}(\tau)^{\beta / \tau} \rightarrow 0
$$

where $\mathscr{T}_{W}$ is of the form

$$
\mathscr{T}_{W}(\tau) \equiv e^{-\frac{\tau}{2} V} e^{\tau A_{\tau}} e^{-\frac{\tau}{2} V}
$$

and $e^{\tau A_{\tau}}$ is a convolution operator with kernel

$$
\frac{1}{N_{\tau}} \exp \frac{1}{\tau} \sum_{\langle x y\rangle} \operatorname{Re} \chi\left(g_{x y}\right),
$$

and the normalization $N_{\tau}$ is chosen to make $\int d g e^{\tau A_{\tau}}(g, \underset{\sim}{h})=1$.

We first want to show that it is enough to prove $\left(\right.$ A.1 $\left.^{1} \tilde{5}\right)$ for $V=0$. To this end we expand in $V$ as before:

$$
\begin{aligned}
\operatorname{Tr} \mathscr{T}_{W}(\tau)^{\beta / \tau} & =\sum_{k \geqq 0} \frac{1}{k !} \sum_{\Sigma k_{i}=k} \frac{k !}{k_{1} ! \ldots k_{N} !} \operatorname{Tr} \prod_{i=1}^{N}\left((-\tau V)^{k_{2}} e^{\tau A_{\tau}}\right) \\
& =\sum_{h \geqq 0} \frac{1}{k !} \int_{0}^{\beta} d t_{1} \ldots d t_{k} \operatorname{Tr}\left(T \prod_{i=1}^{k}\left(-V_{W}\left(\tau\left[\frac{t_{i}}{\tau}\right]\right)\right)\right) e^{\beta A_{\tau}} .
\end{aligned}
$$

Here

$$
V_{W}(t)=e^{t A_{\tau}} V e^{-t A_{\tau}}
$$

Assume that (A.15) holds for $V=0$. Then $\operatorname{Tr} e^{\beta A_{\tau}}$ is bounded uniformly in $\tau$ and the $k^{\text {th }}$ term of (A.17) can again be estimated by const $\beta^{k}\|V\|_{\infty}^{k} \frac{1}{k !}$ (see above). So one only has to prove convergence term by term and only for the difference of the integrands of (A.17) and (A.7). This means we have to analyze terms of the form

$$
\operatorname{Tr} \prod_{i=1}^{k} V e^{s_{i} A_{\tau}}-\operatorname{Tr} \prod_{i=1}^{k} V e^{\frac{1}{2} s_{i} \Delta}
$$

with $0<s_{1}, \ldots, s_{k} \leqq \beta, \sum s_{i}=\beta$. Expanding (A.19) in powers of

$$
R(s) \equiv e^{s A_{\tau}}-e^{\frac{1}{2} s \Delta}
$$


and using Hölder's inequality we obtain the bound

$$
\begin{gathered}
\sum_{n=1}^{k} \sum_{\substack{K \subset\{1, \ldots, k\} \\
|K|=n}}\|V\|_{\infty}^{k} \prod_{i \in K}\left\|R\left(s_{i}\right)\right\|_{\beta / s_{2}} \prod_{i \notin K}\left\|e^{\frac{1}{2} s_{i} \Delta}\right\|_{\beta / s_{i}} \\
\leqq\|V\|_{\infty}^{k}\left\|e^{\frac{1}{2} \beta \Delta}\right\|_{1} \sum_{n=1}^{k}\left(\begin{array}{l}
k \\
n
\end{array}\right)\left(\max _{j}\left\|R\left(s_{j}\right)\right\|_{\beta / s_{j}}\right)^{n} \\
=\|V\|_{\infty}^{k}\left\|e^{\frac{1}{2} \beta \Delta}\right\|_{1}\left\{\left(\max _{1}\left\|R\left(s_{i}\right)\right\|_{\beta / s_{i}}+1\right)^{k}-1\right\},
\end{gathered}
$$

where in the first inequality we used that

$$
\begin{aligned}
\prod_{i \notin K}\left\|e^{\frac{1}{2} s_{i} \Delta}\right\|_{\beta / s_{i}} & =\prod_{i \notin K}\left\|e^{\frac{1}{2} \beta \Delta}\right\|_{1}^{s_{i} / \beta} \\
& \leqq \prod_{i=1}^{k}\left\|e^{\frac{1}{2} \beta \Delta}\right\|_{1}^{s_{1} / \beta}=\left\|e^{\frac{1}{2} \beta \Delta}\right\|_{1}
\end{aligned}
$$

because $\left\|e^{\frac{1}{2} \beta \Delta}\right\|_{1} \geqq 1$.

By our assumption of convergence for $V=0$

$$
\left\|e^{s A_{\tau}}\right\|_{\beta / s} \rightarrow\left\|e^{\frac{1}{2} s \Delta}\right\|_{\beta / s}, \quad(\tau \rightarrow 0)
$$

and hence by Grümm's theorem

$$
\|R(s)\|_{\beta / s} \rightarrow 0 \text {. }
$$

This shows that (A.21) goes to zero and therefore (A.15) holds in general if it holds for $V=0$.

We formulate as a theorem what remains to be proven because it seems to be a slightly nontrivial fact:

Theorem A.2. Let $G$ be a compact Lie group and let $e^{\tau A_{\tau}}$ be a convolution operator on $L^{2}(G, d g)$ given by its kernel

$$
e^{\tau A_{\tau}}(g, h)=\frac{1}{N_{\tau}} e^{\frac{1}{\tau} \operatorname{Re} \chi\left(g h^{-1}\right)},
$$

where $\chi$ is the character of a faithful representation of $G$ and $N_{\tau}=\int d g e^{\tau A_{\tau}}(g, h)$. Then

$$
\operatorname{Tr}\left(e^{\beta A_{\tau}}-e^{\frac{1}{2} \beta \Delta}\right) \rightarrow 0 \quad(\tau \rightarrow 0),
$$

where $\Delta$ is the Laplace-Beltrami operator on $G$ corresponding to the metric induced by $\chi$.

Remarks. 1. This theorem can be viewed as a strong version of a central limit theorem for $G$-valued random variables. In fact some elements of the proof are inspired by the proof of the local version of the central limit theorem (for real valued random variables). 
2. By the Perron-Frobenius theorem [43] $e^{\tau A_{\tau}}$ has a unique positive eigenfunction $\Psi_{0}$ to the eigenvalue $\left\|e^{\tau A_{\tau}}\right\|$. Since the function $\mathbb{1}$ is an eigenfunction with eigenvalue 1 we conclude $\Psi_{0}=\mathbb{1}$ and $\left\|e^{\tau A_{\tau}}\right\|=1$.

3. To apply the theorem to our problem we may either interprete $G$ in the statement as the underlying gauge group because for $V=0$ all the links are uncoupled and it suffices to prove (A.15) for one link or alternatively we may think of $G$ as (gauge group) ${ }^{\text {number of links in } \Lambda_{0}}$.

Proof of Theorem A.2. We diagonalize $\exp \tau A_{\tau}$ and $\Delta$ jointly by Fourier transformation on $G$. Let $q$ run through the inequivalent irreducible unitary representations of $G$. Then $\exp \tau A_{\tau}$ has the eigenvalues $\lambda_{q} / \lambda_{0}$, where

$$
\lambda_{q} \equiv \frac{1}{d_{q}} \int \overline{\chi_{q}(g)} e^{x \operatorname{Re} \chi(g)} d g,
$$

with

$$
x \equiv \frac{1}{\tau}, d_{q} \equiv \chi_{q}(\mathbb{1})
$$

$\chi_{q}$ is the character of the representation $q$ and the subscript 0 refers to the trivial representation.

The eigenvalues of $\Delta$ are given by the (generalized) Casimir elements

$$
C_{q} \equiv \operatorname{Tr} L_{a}^{(q)} L_{a}^{(q)},
$$

where $L_{a}^{(q)}$ is the representative of $L_{a}$ in the representation $q$; as announced we will drop the superscript $q$ in the representation that occurs in the action. To appreciate (A.25) one should recall the orthonormality convention (A.1); the term "generalized" refers to the fact that we are not necessarily using the Killing metric.

Next we want to explain our strategy to prove Theorem A.2. We first prove convergence of the eigenvalues of $\exp \tau A_{\tau}$ to the eigenvalues of $\exp \frac{\tau}{2} \Delta$; this amounts to showing strong convergence which was proven already by Gawejdzi [19], but we will actually need and prove a more detailed statement controlling the speed of convergence (Lemma A.3). The key is, however, again the approximately Gaussian behavior of (A.22) for small $\tau$.

Then we have to control the "tails" in the following sense: Let $S$ be some finite set of irreducible representations of $G$. Then

$$
\begin{aligned}
\sum_{q} d_{q}^{2}\left|\left(\frac{\lambda_{q}}{\lambda_{0}}\right)^{\beta x}-e^{-\frac{1}{2} \beta C_{q}}\right| \leqq & \sum_{q \in S} d_{q}^{2}\left|\left(\frac{\lambda_{q}}{\lambda_{0}}\right)^{\beta x}-e^{-\frac{1}{2} \beta C_{q}}\right| \\
& +\sum_{q \notin S} d_{q}^{2}\left(\frac{\lambda_{q}}{\lambda_{0}}\right)^{\beta x}+\sum_{q \notin S} d_{q}^{2} e^{-\frac{1}{2} \beta C_{q}} .
\end{aligned}
$$

We will need the following estimate for the last two terms: For any $\varepsilon>0$ we have to be able to find a finite set $S$ such that

$$
\sum_{q \notin S} d_{q}^{2}\left(\frac{\lambda_{q}}{\lambda_{0}}\right)^{\beta x}+\sum_{q \notin S} d_{q}^{2} e^{-\frac{1}{2} \beta C_{q}}<\varepsilon
$$



uniformly for large $x$. Since the decay properties of $\exp \left(-\frac{\beta}{2} C_{q}\right)$ are evident we
only have to deal with

$$
\sum_{q \notin S} d_{q}^{2}\left(\frac{\lambda_{q}}{\lambda_{0}}\right)^{\beta x}
$$

We find it necessary to break up the sum (A.27) into three regions:

(I) $C_{q} \leqq x$

(II) $x<C_{q} \leqq x^{1+\varepsilon}$ with some $0<\varepsilon<1$,

(III) $C_{q}>x^{1+\varepsilon}$.

These three regions are controlled in rather different ways; the necessary estimates are contained in Corollary A.4 and Lemmas A.5 and A.6.

But first let us study the convergence of the individual eigenvalues. By Gaussian approximation $\lambda_{q} / \lambda_{0}$ will be approximated by

$$
\begin{aligned}
\tilde{\lambda}_{q} & \equiv \frac{1}{d q} \int_{\mathbb{R}^{p}} \operatorname{Tr} \cos L_{a}^{(q)} t_{a} d \mu_{\tau}(t) \\
& =\frac{1}{d q} \int_{\mathbb{R}^{p}} \operatorname{Tr} e^{i L^{(q)} t_{a}} d \mu_{\tau}(t),
\end{aligned}
$$

where we used the summation convention $L_{a}^{(q)} t_{a} \equiv \sum L_{a}^{(q)} t_{a} ; d \mu_{\tau}$ is the normalized Gaussian measure on $\mathbb{R}^{p}$ with covariance $\tau=1 / x$ :

$$
d \mu_{\tau}(t)=(2 \pi \tau)^{-p / 2} e^{-\frac{t^{2}}{2 \tau}} d^{p} t
$$

and $p$ is the dimension of $G$.

We will prove

\section{Lemma A.3.}

(1) $\left|\frac{\lambda_{q}}{\lambda_{0}}-\tilde{\lambda}_{q}\right| \leqq \operatorname{const} C_{q} x^{-2}$,

(2) $1-\frac{C_{q}}{2 x} \leqq \tilde{\lambda}_{q} \leqq 1-\frac{C_{q}}{2 x}+\frac{C_{q}^{2}}{8 x^{2}}$.

Proof. We first consider (2). We use the elementary inequalities

$$
\begin{aligned}
1-\frac{1}{2}\left(L_{a}^{(q)} t_{a}\right)^{2} & \leqq \cos L_{a}^{(q)} t_{a} \\
& \leqq 1-\frac{1}{2}\left(L_{a}^{(q)} t_{a}\right)^{2}+\frac{1}{4 !}\left(L_{a}^{(q)} t_{a}\right)^{4},
\end{aligned}
$$

which follow by the spectral theorem from the corresponding numerical inequalities (remember that the $L_{a}^{(q)}$ are self-adjoint matrices). If we insert (A.30) in (A.28) the corresponding Gaussian integrals can be calculated. Using

$$
\operatorname{Tr} L_{a}^{(q)} L_{b}^{(q)} L_{a}^{(q)} L_{b}^{(q)} \leqq \operatorname{Tr}\left(L_{a}^{(q)} L_{a}^{(q)}\right)^{2}=\operatorname{Tr} C_{q}^{2}=d_{q} C_{q}^{2},
$$

one obtains (2). 
(1) is also easy: Using the coordinates given by the exponential map in a neighborhood $K_{\varepsilon}$ of the identity $\mathbb{1} \in G$ we see that

$$
\begin{aligned}
\lambda_{q}= & \frac{1}{d_{q}} \int_{\widetilde{K}_{\varepsilon}} \operatorname{Tr} \cos L_{a}^{(q)} t_{a} e^{x \operatorname{Tr} \cos L_{a} t_{a}} d \tilde{g}(t) \\
& +O\left(e^{-c x}\right) e^{x \chi(\mathbb{1})}
\end{aligned}
$$

where $\tilde{K}_{\varepsilon}$ is the inverse image of $K_{\varepsilon}$ under the exponential map and will be assumed to be a ball of radius $\varepsilon . d \tilde{g}(t)$ is the pullback of the Haar measure and

$$
d \tilde{g}(t)=\left(1+O\left(|t|^{2}\right)\right) d^{p} t .
$$

Taylor's formula with remainder allows us to write

where

$$
\begin{aligned}
e^{x \operatorname{Tr} \cos L_{a} t_{a}} & =e^{x \chi(\mathbb{1})-\frac{x}{2}|t|^{2}} e^{Y(t)} \\
& =e^{x \chi(\mathbb{1})-\frac{x}{2}|t|^{2}}\left(1+Y(t) \int_{0}^{1} d s e^{s Y(t)}\right),
\end{aligned}
$$

and

$$
Y(t) \equiv x \operatorname{Tr}\left(\cos L_{a} t_{a}-1\right)+\frac{x}{2}|t|^{2}
$$

$$
\begin{aligned}
0 & \leqq Y(t) \leqq \frac{x}{4 !} \operatorname{Tr}\left(L_{a} t_{a}\right)^{4} \\
& \leqq \frac{x}{4 !}\left(\operatorname{Tr}\left(L_{a} t_{a}\right)^{2}\right)^{2}=\frac{x}{4 !}|t|^{4} .
\end{aligned}
$$

Inserting this in (A.31) gives

$$
\begin{aligned}
\lambda_{0}-\lambda_{q}= & \frac{1}{d_{q}} \int_{\widetilde{K}(\varepsilon)} \operatorname{Tr}\left(\mathbb{1}^{(q)}-\cos L_{a}^{(q)} t_{a}\right) \\
& \cdot e^{x\left(\chi(\mathbb{1})-\frac{1}{2}|t|^{2}\right)} d^{p} t+e^{x \chi(\mathbb{1})}\left(R_{1}+O\left(e^{-c x}\right)\right) \\
= & \frac{1}{d_{q}} \int_{\mathbb{R}^{p}} \operatorname{Tr}\left(\mathbb{1}^{(q)}-\cos L_{a}^{(q)} t_{a}\right) e^{x\left(\chi(\mathbb{1})-\frac{1}{2}|t|^{2}\right)} d^{p} t \\
& +e^{x \chi(\mathbb{1})}\left(R_{1}+O\left(e^{-c x}\right)\right),
\end{aligned}
$$

where with $\lambda=1-2 \varepsilon^{2} / 4$ !

$$
\begin{aligned}
\left|R_{1}\right| \leqq & \frac{1}{d_{q}} \int_{\mathbb{R}^{p}} \operatorname{Tr}\left(\mathbb{1}^{(q)}-\cos L_{a}^{(q)} t_{a}\right) \\
& \cdot\left(\frac{x}{4 !}|t|^{4}+O\left(|t|^{2}\right)\right) e^{-\frac{\lambda x}{2}|t|^{2}} d^{p} t \\
\leqq & \frac{C_{q}}{2} \int_{\mathbb{R}^{p}}\left(\frac{x}{4 !}|t|^{6}+O\left(|t|^{4}\right)\right) e^{-\frac{\lambda x}{2}|t|^{2}} d^{p} t \\
\leqq & N \operatorname{const} \frac{C_{q}}{x^{2}} e^{-x \chi(\mathbb{1})}
\end{aligned}
$$


where $N=\int e^{-\frac{x}{2}|t|^{2}} d^{p} t e^{x \chi(1)}$, and we used (A.30) once more. Similarly one obtains $\lambda_{0}=N\left(1+O\left(\frac{1}{x}\right)\right)$. So we end up with

$$
\begin{aligned}
\frac{\lambda_{q}}{\lambda_{0}}-1 & =\frac{N \tilde{\lambda}_{q}-N}{\lambda_{0}}+O\left(\frac{C_{q}}{x^{2}}\right) \\
& =\left(\tilde{\lambda}_{q}-1\right)\left(1+O\left(\frac{1}{x}\right)\right)+O\left(\frac{C_{q}}{x^{2}}\right) \\
& =\tilde{\lambda}_{q}-1+\left(\tilde{\lambda}_{q}-1\right) O\left(\frac{1}{x}\right)+O\left(\frac{C_{q}}{x^{2}}\right) \\
& =\tilde{\lambda}_{q}-1+O\left(\frac{C_{q}}{x^{2}}\right),
\end{aligned}
$$

where in the last step we used the already proven fact (2). This proves (1).

Lemma A. 3 contains a bit more than strong convergence as can be seen by the following corollary:

\section{Corollary A.4.}

(1) $\left|\frac{\lambda_{q}}{\lambda_{0}}-e^{-C_{q} / 2 x}\right| \leqq \operatorname{const} \frac{C_{q}^{2}}{x^{2}}$.

(2) For $C_{q} \leqq x$

$$
\lambda_{q} / \lambda_{0} \leqq \exp \left(-\frac{3 C_{q}}{8 x}+\frac{b}{x}\right)
$$

where $b$ is some constant.

Proof. (1) is by now obvious. (2) can be seen as follows: By Lemma A.3, (1) and (2)

$$
\begin{aligned}
\frac{\lambda_{q}}{\lambda_{0}} & \leqq \tilde{\lambda}_{q}+\text { const } C_{q} x^{-2} \\
& \leqq \text { const } C_{q} x^{-2}+1-\frac{3}{8} C_{q} x^{-1} \leqq \frac{\text { const }}{x}+e^{-\frac{3 C_{q}}{8 x}} \\
& \leqq \exp \left(-\frac{3 C_{q}}{8 x}\right)\left(1+\frac{\text { const }}{x} \exp \frac{3 C_{q}}{8 x}\right) \\
& \leqq \exp \left(-\frac{3 C_{q}}{8 x}\right)\left(1+\frac{b}{x}\right) \\
& \leqq \exp \left(-\frac{3 C_{q}}{8 x}+\frac{b}{x}\right)
\end{aligned}
$$

where $b=$ const $\exp (3 / 8)$.

Corollary A.4 allows us to control the sum (A.27) in region (I):

$$
\sum_{\substack{q: C_{q} \leqq x \\ q \notin S}} d_{q}^{2}\left(\frac{\lambda_{q}}{\lambda_{0}}\right)^{\beta x} \leqq e^{\beta b} \sum_{q \neq S} d_{q}^{2} e^{-\frac{3 \beta}{8} C_{q}} .
$$


This can be made arbitrarily small by choosing $S$ large enough because

$$
\sum_{q} d_{q}^{2} \exp \left(-\frac{3 \beta}{8} C_{q}\right)=e^{\frac{3 \beta}{8} \Delta}(\mathbb{1}, \mathbb{1})<\infty .
$$

So we now turn to region (II): $x<C_{q} \leqq x^{1+\varepsilon}$. We will use

Lemma A.5. For $C_{q}>x$

$$
\left|\tilde{\lambda}_{q}\right| \leqq \exp \left(-\frac{1}{2 p x} C_{q}\right)+\operatorname{const} \frac{C_{q}^{1 / 2}}{x} .
$$

Proof. Recall that

$$
\begin{aligned}
\tilde{\lambda}_{q} & =\frac{1}{d_{q}} \int_{\mathbb{R}^{p}} d \mu_{\tau}(t) \operatorname{Tr} e^{i L_{a}^{(q)} t_{a}} \\
& =\frac{1}{d_{q}} \int_{\mathbb{R}^{p}} d \mu_{1}(t) \operatorname{Tr} \cos \frac{L_{a}^{(q)} t_{a}}{\sqrt{x}} .
\end{aligned}
$$

We claim that

$$
A_{q} \equiv \int_{\mathbb{R}^{p}} d \mu_{1}(t) \exp \left(\frac{i L_{a}^{(q)} t_{a}}{\sqrt{x}}\right)
$$

is a multiple of the unit matrix $\mathbb{1}^{(q)}$. This is a consequence of the $O(p)$ invariance of the measure $d \mu$ :

$$
\begin{aligned}
& e^{i s_{b} L_{b}^{L q)}} A_{q} e^{-i s_{b} L_{b}^{(q)}} \\
& =\int_{\mathbb{R}^{p}} d \mu_{\tau}(t) e^{i t_{a} O_{a b} L_{b}^{(q)}} \\
& =\int_{\mathbb{R}^{p}} d \mu_{\tau}(t) e^{i t_{a} L_{a}^{(q)}}=A_{q},
\end{aligned}
$$

where $O_{a b}$ is an orthogonal matrix (the adjoint action of $G$ on $\mathfrak{g}$ is orthogonal because we chose an invariant metric on $\mathrm{g}$ ). Because $q$ is an irreducible representation we conclude $A_{q}=\tilde{\lambda}_{q} \mathbb{1}^{(q)}$.

This means that for any normalized vector $\Psi$

$$
\tilde{\lambda}_{q}=\left(\Psi, A_{q} \Psi\right) \text {. }
$$

We will choose $\Psi$ as follows: Because $\sum\left(L_{a}^{(q)}\right)^{2}=C_{q}$, there is at least one $L_{a}^{(q)}$, say $L_{1}^{(q)}$, such that

$$
\left\|L_{1}^{(q)}\right\|^{2} \geqq \frac{1}{p} C_{q}
$$

We will choose $\Psi$ such that

$$
\left(\Psi,\left(L_{1}^{(q)}\right)^{2} \Psi\right)=\left\|L_{1}^{(q)}\right\|^{2} \geqq \frac{1}{p} C_{q} .
$$

Now note that

$$
e^{i L_{a}^{(q)} t_{a}}=\left(\prod_{a} e^{i L_{a}^{(q)} t_{a}}\right) e^{i f\left(b(t) L_{b}^{(q)}\right.}
$$


where in the product the factors are ordered from left to right (the summation convention is obviously lifted) and $f_{b}$ is independent of $q$.

The functions $f_{b}(t)$ are analytic in a disc

$$
D_{\delta} \equiv\left\{t \in \mathbb{R}^{p}|| t \mid<\delta\right\},
$$

and by the Baker-Campbell-Hausdorff formula

$$
f_{b}(t)=O\left(|t|^{2}\right), \quad b=1, \ldots, p
$$

(see [56] for a detailed discussion). This means that

$$
e^{i f_{b}(t) L_{b}^{(q)}}=\mathbb{1}^{(q)}+B^{(q)}(t)
$$

where

$$
\left\|B^{(q)}(t)\right\| \leqq \mathrm{const}|t|^{2} \sup _{a}\left\|L_{a}^{(q)}\right\| \leqq \mathrm{const}|t|^{2} C_{q}^{1 / 2} .
$$

Using (A.38) and (A.36) we obtain

$$
\begin{aligned}
\left|\tilde{\lambda}_{q}\right|= & \left|\int\left(\Psi, e^{i L_{a}^{(q)} t_{a}} \Psi\right) d \mu_{\tau}(t)\right| \\
\leqq & \int\left(\Psi, \prod_{a=1}^{p} e^{i L_{a}^{(q)} t_{a}} \Psi\right) d \mu_{\tau}(t) \\
& +\operatorname{const} C_{q}^{1 / 2} \int|t|^{2} d \mu_{\tau}(t) \\
= & \left(\Psi, \prod_{a=1}^{p} \exp \left(-\frac{\tau}{2}\left(L_{a}^{(q)}\right)^{2}\right) \Psi\right)+\operatorname{const} \tau C_{q}^{1 / 2} \\
\leqq & \exp \left(-\frac{\tau}{2 p} C_{q}\right)+\operatorname{const} C_{q}^{1 / 2} \tau
\end{aligned}
$$

as claimed.

Lemma A.5 obviously implies that for $C_{q} \leqq x^{1+\varepsilon}$ and $x$ large enough there is a constant $\tilde{\varrho}<1$ such that for $C_{q}>x$

$$
\left|\tilde{\lambda}_{q}\right|<\tilde{\varrho}
$$

Using Lemma A.3 we obtain in region (II) $\left(x<C_{q} \leqq x^{1+\varepsilon}\right)$ :

$$
\frac{\lambda_{q}}{\lambda_{0}} \leqq\left|\tilde{\lambda}_{q}\right|+\text { const } \frac{C_{q}}{x^{2}} \leqq \tilde{\varrho}+\text { const } \frac{C_{q}}{x^{2}},
$$

and again by choosing $x$ large enough we can find a $c>0$ such that

$$
\frac{\lambda_{q}}{\lambda_{0}} \leqq e^{-c}<1
$$

in region (II). This will control region (II) because

$$
\sum_{x<C_{q} \leqq x^{1+\varepsilon}} d_{q}^{2} \leqq \operatorname{const} x^{\frac{p}{2}(1+\varepsilon)} .
$$


To see (A.42) note that

$$
\sum_{q \in \hat{G}} d_{q}^{2} e^{-t C_{q}}=e^{t \Delta}(\mathbb{1}, \mathbb{1})=O\left(t^{-\frac{p}{2}}\right)
$$

and therefore

$$
\begin{aligned}
\sum_{C_{q} \leqq x^{1+\varepsilon}} d_{q}^{2} \leqq e^{t x^{1+\varepsilon}} \sum_{q \in \hat{G}} e^{-t C_{q}} d_{q}^{2} \\
\leqq \text { const } t^{-\frac{p}{2}} e^{t x^{1+\varepsilon}} .
\end{aligned}
$$

Putting $t=x^{-1-\varepsilon}$ proves (A.42). From (A.41) and (A.42) we obtain

$$
\sum_{x<C_{q} \leqq x^{1+\varepsilon}}\left(\frac{\lambda_{q}}{\lambda_{0}}\right)^{\beta x} d_{q}^{2} \leqq e^{-\beta c x} x^{\frac{p}{2}(1+\varepsilon)} \text { const } \rightarrow 0 \quad(\tau \rightarrow 0) .
$$

This leaves us with the region (III): $C_{q}>x^{1+\varepsilon}, \varepsilon<1$. To control this region we will exploit smoothness properties of the kernel of $e^{\tau A_{\tau}}$ which will give decay properties of the Fourier coefficients $\lambda_{q}$.

Lemma A.6. (1) Let $n \geqq 0$ be an integer. Then

$$
\frac{1}{n !} \sum_{q} d_{q}^{2} \lambda_{q}^{2} C_{q}^{n / 2} \leqq c^{n} x^{n / 2} \sum_{q} d_{q}^{2} \lambda_{q}^{2}
$$

with some constant $c$.

(2) Let $t<c^{-1}$. Then

$$
\sum_{q} d_{q}^{2} \lambda_{q}^{2} \exp \left(t \sqrt{\frac{C_{q}}{x}}\right) \leqq \frac{1}{1-c t} \sum d_{q}^{2} \lambda_{q}^{2}
$$

Proof. (2) is an easy consequence of (1). So we have to estimate

$$
\sum_{q} d_{q}^{2} \lambda_{q}^{2} C_{q}^{n / 2}=\int e^{x \operatorname{Re} \chi(g)}(-\Delta)^{n / 2} e^{x \operatorname{Re} \chi(g)} d g .
$$

$-\Delta$ can be expressed in terms of the standard left invariant vector fields $X_{a}$ defined by

$$
\left.\left(X_{a} f\right)(g) \equiv \frac{d}{i d t} f\left(g e^{i t_{a} L_{a}}\right)\right|_{t=0},
$$

as

$$
-\Delta=\sum_{a} X_{a} X_{a}
$$

Note the following simple facts

$$
\begin{gathered}
\left|\prod_{i=1}^{2 k}\left(X_{b_{\imath}} \operatorname{Re} \chi\right)\left(e^{i L_{a} t_{a}}\right)\right| \leqq\left(c|t|^{2}\right)^{k}, \\
\left|\left(X_{b_{1}} \ldots X_{b_{2 k}} \operatorname{Re} \chi\right)(g)\right| \leqq c^{k}
\end{gathered}
$$


Using (A.48) in conjunction with (A.30) we obtain after the introduction of local coordinates on $G$ by the exponential map

$$
\begin{aligned}
& \left|\int e^{2 x \operatorname{Re} \chi(g)} \prod_{i=1}^{2 k}\left(X_{b_{i}} \operatorname{Re} \chi\right)(g) d g\right| \\
& \leqq e^{2 x \chi(\mathbb{1})} \int_{|t| \leqq \varepsilon} d^{p} t e^{-\lambda x|t|^{2}}\left(c|t|^{2}\right)^{k} \\
& \leqq e^{2 x \chi(\mathbb{1})} \int_{\mathbb{R}^{p}} d^{p} t e^{-\lambda x|t|^{2}}\left(c|t|^{2}\right)^{k} \\
& \quad \leqq N\left(\frac{c^{\prime}}{x}\right)^{k}(2 k-1) ! !
\end{aligned}
$$

where $\lambda=1-2 \varepsilon^{2} / 4$ ! and

$$
\begin{aligned}
N & =\text { const } e^{2 x \chi(\mathbb{1})} \int d^{p} t e^{-x|t|^{2}} \\
& =\text { const } \int e^{2 x \operatorname{Re} \chi(g)} d g\left(1+O\left(\frac{1}{x}\right)\right) .
\end{aligned}
$$

So we only have to analyze the possible terms arising from $X_{b_{1}} \ldots X_{b_{2 k}} e^{x \operatorname{Re} \chi}$ by Leibniz's rule and estimate their number. There is one term of the form

$$
x^{2 k} \prod_{i=1}^{2 k}\left(X_{b_{i}} \operatorname{Re} \chi\right) e^{x \operatorname{Re} \chi}
$$

which when inserted in (A.50) gives

$$
N\left(c^{\prime} x\right)^{k}(2 k-1) ! !
$$

Then there is a term of the form

$$
\left|x^{2 k-1} e^{x \operatorname{Re} \chi} X_{b_{1}}\left(\prod_{i=2}^{2 k}\left(X_{b_{l}} \operatorname{Re} \chi\right)\right)\right| \leqq \sum_{j=1}^{2 k-1}\left|c x^{2 k-1} e^{x \operatorname{Re} \chi} \prod_{\substack{2 \leqq i \leqq 2 k \\ i \neq j}}\left(X_{b_{i}} \operatorname{Re} \chi\right)\right|,
$$

which after insertion in (A.50) gives

$$
N\left(c^{\prime} x\right)^{k}(2 k-1)(2 k-3) ! !
$$

etc. Finally we reach

$$
N\left(c^{\prime} x\right)^{k}(2 k-k+1)^{k-1}(2 k+1-2 k) ! ! .
$$

The other terms do not involve integrals other than the normalization $N$ and can be bounded collectively by

$$
\sum_{l=k}^{2 k}\left(c^{\prime} x\right)^{k}(2 k-l)^{l} N \leqq\left(c^{\prime} x\right)^{k}(2 k) ! N \leqq \frac{N}{2}\left(2 c^{\prime} x\right)^{k}(2 k) !
$$

(for $k>0$ ). 
Summing up the terms from (A.51) to (A.52) we obtain

$$
\begin{aligned}
& N\left(c^{\prime} x\right)^{k} \sum_{l=0}^{k-1}(2 k-l)^{l}(2 k-1-2 l) ! ! \\
& \leqq\left(c^{\prime} x\right)^{k}(2 k) ! N \leqq \frac{N}{2}\left(2 c^{\prime} x\right)^{k}(2 k) !
\end{aligned}
$$

(for $k>0$ ).

So we finally end up with

$$
\begin{aligned}
& \int e^{x \operatorname{Re} \chi}(-\Delta)^{k} e^{x \operatorname{Re} \chi} d g \\
& \quad=\int e^{x \operatorname{Re} \chi} \prod_{i=1}^{k}\left(X_{a_{i}} X_{a_{i}}\right) e^{x \operatorname{Re} \chi} d g \\
& \quad \leqq p^{k}(\text { const })^{k}(2 k) ! \int e^{2 x \operatorname{Re} \chi(g)} d g\left(1+O\left(\frac{1}{x}\right)\right)
\end{aligned}
$$

for $k>0$. We may absorb the $O\left(\frac{1}{x}\right)$ in $(\text { const })^{k}$; so $(1)$ is proven for $n=2 k$. For $n=2 k+1$ the Schwarz inequality completes the job.

Lemma A.6 can now be used to complete the proof of Theorem (A.2) by estimating the "tail" (A.27) in region (III): Chose some $t>c^{-1}$ and define $c^{\prime}=t / 3$. Then for $C_{q}>x^{1+\varepsilon}$

$$
\begin{aligned}
d_{q}^{2}\left(\frac{\lambda_{q}}{\lambda_{0}}\right)^{2} & \leqq \text { const } \exp \left(-3 c^{\prime} \sqrt{C_{q} / x}\right) \frac{\int e^{2 x \operatorname{Re} x(g)} d g}{\left(\int e^{x \operatorname{Re} x(g)} d g\right)^{2}} \\
& \leqq \text { const } x^{p / 2} \exp \left(-2 c^{\prime} x^{\varepsilon / 2}\right) \exp \left(-c^{\prime} \sqrt{C_{q} / x}\right) \\
& \leqq c(\varepsilon) \exp \left(-c^{\prime} x^{\varepsilon / 2}\right) \exp \left(-c^{\prime} \sqrt{C_{q} / x}\right)
\end{aligned}
$$

where $c(\varepsilon)$ is a constant depending on $\varepsilon$. So for $\varepsilon>0, x>x_{0}(\varepsilon), C_{q}>x^{1+\varepsilon}$ we get

$$
d_{q}^{2}\left(\frac{\lambda_{q}}{\lambda_{0}}\right)^{2} \leqq e^{-c^{\prime} \sqrt{C_{q} / x}}
$$

and therefore

$$
\sum_{C_{q}>x^{1+\varepsilon}} d_{q}^{2}\left(\frac{\lambda_{q}}{\lambda_{0}}\right)^{\beta x} \leqq \sum_{C_{q}>x^{1+\varepsilon}} e^{-c^{\prime} \beta \sqrt{C_{q} x}} .
$$

If we recall that by (A.42) the number of representations $q$ obeying $K-1<C_{q} \leqq K$ is bounded by const $K^{p / 2}$ we see that the right hand side of (A.54) goes to zero as $x \rightarrow \infty$. This completes the control of the tails and Theorem A.2 is now proven.

\section{Appendix B}

In this appendix we want to adapt the random walk expansion of [15] to lattice Yang-Mills theory at finite temperature. We first decompose our model of $L_{0}$ time 
layers into $L_{0}$ coupled nonlinear $\sigma$-models in $d$ dimensions :

$$
G(x-y)=\frac{1}{Z} \sum_{\left\{a_{m}, b_{m}\right\}} \int \prod_{m=1}^{L_{0}} d v_{m} e^{-\tau V\left(y_{m}\right)}\left[u_{m x}^{a_{m} a_{m+}+} \overline{u_{m y}^{b_{m} b_{m}+1}}\right]_{m},
$$

where we used the notation

$$
\begin{gathered}
{[\cdot]_{m}=\int \cdot e^{-S_{m}} d \underline{u},} \\
S_{m}=-J \operatorname{Re} \sum_{\langle x y\rangle} \chi\left(u_{x}^{*} v_{x y}^{m} u_{y} v_{x y}^{m+1}\right) .
\end{gathered}
$$

We first treat the case $G=\mathrm{SU}(2)$ and use the standard identification of SU(2) with $S_{3}$ :

$$
\left.\begin{array}{rl}
u(s) & \equiv s_{0}+i \mathbf{s} \cdot \boldsymbol{\sigma}, \\
d u(s) & \equiv \delta\left(s^{2}-1\right) d^{4} s, \\
\phi & \equiv \frac{\partial}{\partial s_{0}}+i \sum_{k=1}^{3} \sigma_{k} \frac{\partial}{\partial s_{k}},
\end{array}\right\}
$$

where $\sigma_{1}, \sigma_{2}, \sigma_{3}$ are the Pauli matrices. Note that for any $g \in \mathrm{SU}(2)$

$$
\phi \operatorname{tr} u(s)^{*} g=g \text {. }
$$

Our first input is the following

\section{Lemma B.1.}

(i) $u(s) \delta\left(s^{2}-1\right)=-\phi \int_{0}^{\infty} \delta\left(s^{2}-1+\lambda\right) d \lambda$
(ii) $u(s) \int_{0}^{\infty} \frac{\lambda^{n}}{n !} \delta\left(s^{2}-1+\lambda\right) d \lambda=-\phi \int_{0}^{\infty} \frac{\lambda^{n+1}}{(n+1) !} \delta\left(s^{2}-1+\lambda\right) d \lambda$.

Proof. This is a simple computation using

$$
\int_{0}^{\infty} \delta\left(s^{2}-1+\lambda\right) \lambda^{n} d \lambda=\left(1-s^{2}\right)_{+}^{n}
$$

where

$$
\left(1-s^{2}\right)_{+}^{n}= \begin{cases}\left(1-s^{2}\right)^{n} ; & s^{2} \leqq 1 \\ 0 ; & s^{2} \geqq 1 .\end{cases}
$$

We use this to expand $\left[u_{x} \bar{u}_{y}\right]_{m}$. First we use (i) and the property $\not \operatorname{tr} u^{*}(s) g=g$ to obtain

$$
\begin{aligned}
& \int d u_{x} u_{x} \prod_{y^{\prime}:\left|x-y^{\prime}\right|=\varepsilon} e^{J \operatorname{Tr}\left(u_{\bar{x}}^{-1} v_{x x}^{m} y^{\prime} u_{y^{\prime}} v_{y}^{m+1}+1\right)} \\
& =\int \delta\left(s_{x}^{2}-1\right) d^{4} s_{x} u\left(s_{x}\right) \prod_{y^{\prime}:\left|x-y^{\prime}\right|=\varepsilon} e^{J \operatorname{Tr}\left(u_{x}^{*} v_{x}^{m} y^{\prime} u_{y^{\prime}} v_{y}^{m+1}+1\right)} \\
& =\sum_{y^{\prime}:\left|x-y^{\prime}\right|=\varepsilon} J \int_{0}^{\infty} d \lambda_{x} \delta\left(s_{x}^{2}-1+\lambda_{x}\right)\left(v_{x y^{\prime}}^{m} u_{y^{\prime}} v_{y^{\prime} x}^{m+1}\right) \\
& \cdot \prod_{y^{\prime}:|x-y|=\varepsilon} e^{J \operatorname{Tr}\left(u_{\bar{x}}^{1} v_{x y^{\prime}}^{m} u_{y^{\prime}} v_{y^{\prime} x}^{m+1}\right)} .
\end{aligned}
$$


If we continue to integrate by parts using Lemma B.1 we obtain the following expansion of $\left[u_{x} \bar{u}_{y}\right]_{m}$ in a sum over all paths $\omega$ from $x$ to $y$ :

$$
\begin{aligned}
{\left[u_{x}^{k l} \overline{u_{y}^{i j}}\right]_{m}=} & \sum_{\omega: x \rightarrow y} J^{|\omega|} v_{\omega, m}^{k i} v_{\omega-1, m+1}^{l j} \\
& \cdot \int d \prod_{x \in \omega} \frac{\lambda_{x}^{n_{x}(\omega)-1}}{\left(n_{x}(\omega)-1\right) !} \int d u \prod_{x} \delta\left(s_{x}^{2}-1+\lambda_{x}\right) e^{-S_{m}} .
\end{aligned}
$$

Here $n_{x}(\omega)$ is the number of times $\omega$ hits $x,|\omega|=\sum_{x} n_{x}(\omega)-1$, and $v_{\omega, m}=\prod_{\langle x y\rangle \in \omega} v_{x y}^{m}$ (ordered product, $\omega^{-1}$ is obtained from reversing the orientation of $\omega$.

Changing variables in (B.5) we obtain

$$
\begin{aligned}
{\left[u_{x}^{k l} \overline{u_{y}^{i j}}\right]_{m}=} & \sum_{\omega: x \rightarrow y} J^{|\omega|} v_{\omega, m}^{k i} v_{\omega-1}^{l j}, m+1 \\
& \cdot \int d \lambda_{\sim} \prod_{x} \frac{\lambda_{x}^{n_{x}(\omega)-1}}{\left(n_{x}(\omega)-1\right) !} \prod_{x \in \omega}\left(1-\lambda_{x}\right) \tilde{Z}_{m}^{(\lambda)},
\end{aligned}
$$

where $\tilde{Z}_{m}^{(\lambda)}$ is obtained from $\tilde{Z}_{m}^{(0)}=\int e^{-S_{m}} d \underset{\sim}{u}$ by replacing the coupling constant $J$ for the link $\langle x y\rangle$ by

$$
J_{x y}=\sqrt{1-\lambda_{x}} \sqrt{1-\lambda_{y}} J
$$

Note that all integrations over $\lambda$ can be restricted without loss to the interval $[0,1]$ because $\delta\left(s^{2}-1+\lambda\right)=0$ for $\lambda>1$. With the notation

$$
F\left(v_{m}, v_{m+1} \mid \omega\right) \equiv \int d \prod_{x} \frac{\lambda_{x}^{n_{x}(\omega)-1}}{\left(n_{x}(\omega)-1\right) !} \prod_{x \in \omega}\left(1-\lambda_{x}\right) \tilde{Z}_{m}^{(\lambda)} / \tilde{Z}_{m}^{(0)},
$$

we get

$$
\left[u_{x}^{k l} \overline{u_{y}^{i j}}\right]=\sum_{\omega: x \rightarrow y} J^{|\omega|} v_{\omega, m}^{k i} v_{\omega-1, m+1}^{l j} \tilde{Z}_{m}^{(0)} F\left({\underset{\sim}{m}}_{m}, \underline{\sim}_{m+1} \mid \omega\right) .
$$

Inserting this in (B.1) we get our desired expansion of $G(x-y)$ into a sum over ("random") surfaces consisting of vertical plaquettes and spanned between $L_{x}$ and $L_{y}$ :

$$
G(x-y)=\sum_{S: \partial S=\left\{L_{x}, L_{y}\right\}} J^{|S|}\left\langle\prod_{m=1}^{L_{0}} F\left(v_{m}, v_{m+1} \mid \omega_{m}^{S}\right) \chi\left(u_{C_{m}(S)}^{m}\right)\right\rangle,
$$

where $\omega_{m}^{S}$ is the path obtained from $S$ by intersecting it with the $m^{\text {th }}$ time layer, $C_{m}(S)$ is the combined path

$$
\omega_{m}^{S} \circ\left(\omega_{m+1}^{S}\right)^{-1}, \quad|S|=\sum\left|\omega_{m}^{S}\right|
$$

(note that we do not include horizontal parts in this "area").

To see absolute and uniform convergence of this expansion let us apply Hölder's inequality to

$$
\left\langle\prod_{m=1}^{L_{0}} \tilde{Z}_{m}^{\left(\lambda_{m}\right)} / \tilde{Z}_{m}^{(0)} \chi\left(u_{C_{m}(S)}^{m}\right)\right\rangle=\frac{1}{Z} \operatorname{Tr} \prod_{m=1}^{L_{0}} P_{0} \mathscr{T}_{m} \chi\left(u_{C_{m}(S)}\right)
$$


where $\mathscr{T}_{m}$ is the transfer matrix (in 1 direction) obtained by replacing $J$ by $J_{x y}$ according to (B.6). Now note that

$$
\left\|\mathscr{T}^{-1 / 2} \mathscr{T}_{m} \mathscr{T}^{-1 / 2}\right\| \leqq 1,
$$

which follows by differentiating $\left\langle\varphi\left|\mathscr{T}_{m}\right| \varphi\right\rangle$ with respect to $J_{x v}$ and noting that the result is nonnegative by reflection positivity. Applying (B.11) and Hölder's inequality we obtain

$$
\begin{aligned}
\left|\operatorname{Tr} \prod_{m=1}^{L_{0}} P_{0} \mathscr{T}_{m} \chi\left(u_{C_{m}(S)}\right)\right| & =\left|\operatorname{Tr} \prod_{m=1}^{L_{0}} P_{0}\left(\mathscr{T}^{1 / 2} \chi\left(u_{C_{m}(S)}\right) \mathscr{T}^{1 / 2}\right)\left(\mathscr{T}^{-1 / 2} \mathscr{T}_{m} \mathscr{T}^{-1 / 2}\right)\right| \\
& \leqq \prod_{m=1}^{L_{0}}\left\|P_{0} \mathscr{T}^{1 / 2} \chi\left(u_{C_{m}(S)}\right) \mathscr{T}^{1 / 2}\right\|_{L_{0}} \\
& \leqq \chi(\mathbb{1})^{L_{0}}\|\mathscr{T}\|_{L_{0}}^{L_{0}},
\end{aligned}
$$

and therefore [see (B.10)]

$$
\left|\left\langle\prod_{m=1}^{L_{0}} \tilde{Z}_{m}^{\left(\lambda_{m}\right)}\left(\tilde{Z}_{m}^{(0)}\right)^{-1} \chi\left(u_{C_{m}(S)}\right)\right\rangle\right| \leqq \frac{1}{Z} \operatorname{Tr} P_{0} \mathscr{T}^{L_{0}} \chi(\mathbb{1})^{L_{0}}=\chi(\mathbb{1})^{L_{0}} .
$$

Now we can bound (B.9), using the fact that the $\lambda$-integrals contained in the $F$ 's [Eq. (B.7)] can be computed:

$$
G(x-y) \leqq \sum_{S: O S=\left\{L_{x}, L_{y}\right\}} J^{|S|} \chi(\mathbb{1})^{L_{0}} \prod_{m=1}^{L_{0}} a_{n_{x}\left(\omega_{m}\right)},
$$

with

$$
a_{n} \equiv \int_{0}^{1} \frac{\lambda^{n-1}}{(n-1) !}(1-\lambda) d \lambda=\frac{1}{(n+1) !} .
$$

To estimate the sum over random paths we consider all paths starting at $x$ and having at least length $|x-y|$. Note that then there are $2 d$ possibilities to make a path $\omega^{\prime}$ of lengths $|\omega|+1$ from a path $\omega$ of length $|\omega|$ and at least one of them will end in a point $z$ with $n_{z}\left(\omega^{\prime}\right) \geqq 2$. Inserting this in (B.13) we obtain

$$
G(x-y) \leqq\left(\sum_{k \geqq|x-y|} J^{k}\left(\frac{2 d-1}{1+1}+\frac{1}{1+2}\right)^{k} \chi(\mathbb{1})\right)^{L_{0}},
$$

which shows absolute convergence of the expansion and at the same time confinement provided

$$
J<\left(\frac{2 d-1}{2}+\frac{1}{3}\right)^{-1}=\left(d-\frac{1}{6}\right)^{-1} .
$$

Let us now turn to $U(1)$ : Here we may represent the elements of $U(1)$ as unit vectors in $\mathbb{R}^{2}$ and we obtain an expansion like (B.9) but with $J$ replaced by $J / 2$ and $1-\lambda$ replaced by 1 in the definition of $F(\ldots \mid \omega)$ because the change of variables in (B.5) involves one less power of $1-\lambda$. We obtain confinement for

$$
J<2\left(\frac{2 d-1}{1}-\frac{1}{2}\right)^{-1}=\left(d-\frac{1}{4}\right)^{-1} .
$$


Let us finally remark that (B.13) can be improved for $L_{0}>1$. For notational convenience assume $L_{0}=2 N_{0}, N_{0} \in \mathbb{Z}_{+}$. Then

$$
\left\|P_{0} \mathscr{T}^{1 / 2} \chi\left(u_{C_{m}(S)}\right) \mathscr{T}^{1 / 2}\right\|_{L_{0}}
$$

becomes

$$
\begin{aligned}
& \left(\operatorname{Tr}\left(P_{0} \chi\left(u_{C_{m}(S)}\right) \mathscr{T} \overline{\chi\left(u_{C_{m}(S)}\right)} \mathscr{T}\right)^{N_{0}}\right)^{1 / L_{0}} \\
& \quad=Z\left\langle\prod_{n=1}^{N_{0}} \chi\left(u_{C_{m}(S)}^{n}\right) \overline{\chi\left(u_{C_{m}(S)}^{n+1}\right)}\right\rangle^{1 / L_{0}} \\
& \quad \equiv Z \varrho\left(C_{m}(S)\right),
\end{aligned}
$$

which leads to

$$
\left|\left\langle\prod_{m=1}^{L_{0}} F\left(v_{m}, \underline{v}_{m+1} \mid \omega_{m}^{S}\right) \chi\left(u_{C_{m}(S)}^{m}\right)\right\rangle\right| \leqq \prod_{m=1}^{L_{0}}\left[\left(\prod_{x \in \Lambda} \frac{1}{\left(n_{x}\left(\omega_{m}^{S}\right)+1\right) !}\right) \varrho\left(C_{m}(S)\right)\right] .
$$

One could now use the perimeter decay of $\varrho\left(C_{m}(S)\right)$ to sharpen the bounds on $J$ (see [15]) for details.

\section{Appendix C}

The purpose of this appendix is to prove Eq. (III.88):

$$
\sup _{n}\left(\frac{I_{n}(J)^{2}}{I_{n+1}(J) I_{n-1}(J)}-1\right)=\left(\frac{I_{0}(J)}{I_{1}(J)}\right)^{2}-1 .
$$

Here

$$
I_{n}(J)=\sum_{k=0}^{\infty} \frac{1}{k !(k+n) !}\left(\frac{J}{2}\right)^{2 k+n}
$$

is the $n^{\text {th }}$ modified Bessel function. Let us first expand the product of two such functions:

$$
\begin{aligned}
I_{n}(J) I_{m}(J) & =\sum_{k=0}^{\infty}\left(\frac{J}{2}\right)^{2 k+n+m} \sum_{p=0}^{k} \frac{1}{p !(k-p) !(p+m) !(n+k-p) !} \\
& =\sum_{k=0}^{\infty}\left(\frac{J}{2}\right)^{2 k+n+m} \frac{(2 k+n+m) !}{k !(n+k) !(m+k) !(m+n+k) !} .
\end{aligned}
$$

The last equality was obtained by expanding $(1+z)^{2 k+m+n}$ and comparing coefficients with the product of the expansions of $(1+z)^{k+m}$ and $(1+z)^{k+n}$. Equation (C.1) will now follow from

\section{Lemma C.1.}

$$
\left(\frac{I_{0}}{I_{1}}\right)^{2} \geqq \frac{I_{n}^{2}}{I_{n+1} I_{n-1}} .
$$

Proof. We have to show that

$$
\alpha_{n}(J) \equiv I_{0}^{2} I_{n+1} I_{n-1}-I_{1}^{2} I_{n}^{2} \geqq 0 .
$$


For this we use (C.3) to obtain

$$
\begin{aligned}
\alpha_{n}(J)= & \sum_{m=0}^{\infty}\left(\frac{J}{2}\right)^{2 m+2 n} \sum_{p=0}^{m} \frac{(2 p+2 n) !(2 m-2 p) !}{p !(n+p) !^{2}(2 n+p) !(m-p) !^{4}} \frac{n+p}{n+p+1} \\
& -\sum_{m=0}^{\infty}\left(\frac{J}{2}\right)^{2 m+2+2 n} \sum_{p=0}^{m} \frac{(2 p+2 n) !(2 m+2-2 p) !}{p !(n+p) !^{2}(2 n+p) !(m+1-p) !^{4}} \frac{m-p+1}{m-p+2} \\
& \geqq \sum_{m=0}^{\infty}\left(\frac{J}{2}\right)^{2 m+2 n} \sum_{p=0}^{m} \frac{(2 p+2 n) !(2 m-2 p) !}{p !(n+p) !^{2}(2 n+p) !(m-p) !^{4}}\left(\frac{n+p}{n+p+1}-\frac{m-p}{m-p+1}\right),
\end{aligned}
$$

where in the sum corresponding to $I_{n}^{2} I_{1}^{2}$ we changed $m$ to $m-1$. Note that the last bracket in (C.3) is positive for $n+p \geqq m-p$. We may therefore restrict the sum over $m$ to $m \geqq n$ and the sum over $p$ to $p \leqq m-n$ and thus bound (C.3) by

$$
\begin{aligned}
\sum_{m=n}^{\infty}\left(\frac{J}{2}\right)^{2 m+2 n} \sum_{p=0}^{m-n} \frac{(2 p+2 n) !(2 m-2 p) !}{(n+p) !^{2}(m-p) !^{2}} \\
\cdot \frac{1}{p !(2 n+p) !(m-p) !^{2}}\left(\frac{n+p}{n+p+1}-\frac{m-p}{m-p+1}\right) .
\end{aligned}
$$

If we change the summation index $p$ in the second term to $m-n-p$ we see that we only have to show that for $2 p \leqq m-n$

$$
\frac{1}{p !(2 n+p) !(m-p) !^{2}} \leqq \frac{1}{(m-n-p) !(m+n+p) !(n+p) !^{2}},
$$

because the positive term then dominates the negative one and thus $\alpha_{n}(J) \geqq 0$.

So we just need

Lemma C.2. For $0 \leqq p \leqq \frac{m-n}{2}$,

$$
\frac{1}{p !(2 n+p) !((m-p) !)^{2}} \leqq \frac{1}{(m-n-p) !(n+m-p) !((n+p) !)^{2}}
$$

Proof. We proceed inductively. First put $p=\left[\frac{m-n}{2}\right]$. Then the lemma holds because then $p+n=m-p$ (for even $p$ ) or $p+n=m-p-1$ (for odd $p$ ). Replacing now $p$ by $p-1$ the right hand side picks up a factor

$$
\frac{(n+p)^{2}}{(m-n-p)(n+m-p)}=\frac{n^{2}+2 p n+p^{2}}{(m-p)^{2}-n^{2}}
$$

whereas the left hand side picks up the smaller factor

$$
\frac{p(2 n+p)}{(m-p+1)^{2}}
$$




\section{References}

1. de Angelis, G.F., De Falco, D., Guerra, F.: Phys. Rev. D 17, 1624 (1978)

2. Borgs, C.: Freie Quarks bei hoher Temperatur, ein strenger Beweis im Rahmen der Gittereichtheorien. Diplomarbeit, München 1982

3. Borgs, C., Seiler, E.: Nucl. Phys. B 215 [FS 7], 125 (1983)

4. Bricmont, J., Fröhlich, J.: Phys. Lett. 122 B, 73 (1983)

5. Brydges, D.C., Federbush, P.: Commun. Math. Phys. 62, 79 (1978)

6. Brydges, D.C., Federbush, P.: Commun. Math. Phys. 73, 197 (1980)

7. Bunk, B., Wolff, U.: Nucl. Phys. B 215 [FS 7], 495 (1983)

8. Caracciolo, S., Menotti, P.: Ann. Phys. (N.Y.) 122, 74 (1979); Nucl. Phys. B 180 [FS2], 428 (1981)

9. Creutz, M.: Phys. Rev. D 21, 2308 (1979)

10. Creutz, M.: Phys. Rev. D 15, 1128 (1977)

11. Davies, E.B.: J. London Math. Soc. 7, 483 (1973)

12. Dolan, L., Jackiw, R.: Phys. Rev. D 9, 3320 (1974)

13. Drouffe, J.M.: Phys. Rev. D 18, 1174 (1978)

14. Drühl, K., Wagner, H.: Ann. Phys. (N.Y.) 141, 225 (1982)

15. Durhuus, B., Fröhlich, J.: Commun. Math. Phys. 75, 103 (1980)

16. Engels, J., Karsch, F., Satz, H., Montvay, I.: Nucl. Phys. B 205 [FS 5], 545 (1982)

17. Fredenhagen, K.: Structural aspects of gauge theories in the algebraic framework of quantum field theory. Talk presented at the Colloquium in honour of R. Haag's $60^{\text {th }}$ birthday, Freiburg University preprint 1982

18. Fröhlich, J., Simon, B., Spencer, T.: Commun. Math. Phys. 50, 79 (1976)

19. Gawędzki, K.: Phys. Rev. D 26, 3593 (1982)

20. Ginibre, J.: Commun. Math. Phys. 16, 310 (1970)

21. Grümm, H.: Rep. Math. Phys. 14, 211 (1973)

22. Guerra, F., Immirzi, G., Marra, R.: Lett. Nuovo Cimento 23, 237 (1978)

23. Guth, A.: Phys. Rev. D 23, 347 (1981)

24. Hewitt, E., Ross, K.A.: Abstract harmonic analysis, Vols. I, II. Berlin, Heidelberg, New York: Springer 1970

25. Kadanoff, L.: Ann. Phys. 100, 359 (1976)

26. Kogut, J., Susskind, L.: Phys. Rev. D 11, 395 (1975)

27. Kuti, J., Polónyi, P., Szlachanyi, K.: Phys. Lett. 98 B, 199 (1981)

28. Linde, A.D.: Rep. Prog. Phys. 42, 389 (1979)

29. Lüscher, M.: Commun. Math. Phys. 54, 283 (1977)

30. Mack, G., Meyer, H.: Nucl. Phys. B 200 [FS4], 249 (1982)

31. McKean, H.P., Jr.: Stochastic integrals. New Yoek: Academic Press 1969

32. McKean, H.P., Singer, I.M.: J. Diff. Geom. 1, 43 (1967)

33. McLerran, L.D., Svetitsky, B.: Phys. Lett. 98 B, 195 (1981)

34. Migdal, A.A.: Zh. Eksp. Teor. Fiz. 69, 810 (1975) [Sov. Phys. JETP 42, 413 (1976)]

35. Mưnster, G.: Nucl. Phys. B 180 [FS 2], 23 (1981)

36. Nauenberg, M., Toussaint, D.: Nucl. Phys. B 190 [FS 3], 217 (1981)

37. Okubo, S.: J. Math. Phys. 18, 2382 (1977)

38. Osterwalder, K., Seiler, E.: Ann. Phys. (N.Y.) 110, 440 (1978)

39. Patrascioiu, A., Seiler, E., Stamatescu, I.O.: Phys. Lett. 107 B, 364 (1981)

40. Polyakov, A.M.: Phys. Lett 72 B, 477 (1978)

41. Polónyi, P., Szlachanyi, K.: Phys. Lett. 110 B, 395 (1982)

42. Ray, D.B., Singer, I.M.: Adv. Math. 7, 145 (1971)

43. Reed, M., Simon, B.: Methods of modern mathematical physics, Vol. I: Functional analysis. New York: Academic Press 1972; Vol. IV: Analysis of operators. New York: Academic Press 1978

44. Seiler, E.: Phys. Rev. D 18, 482 (1978)

45. Seiler, E.: Gauge theories as a problem of constructive quantum field theory and statistical mechanics. In: Lecture Notes in Physics, Vol. 159. Berlin, Heidelberg, New York: Springer 1982

46. Seiler, E.: Mathematical problems in theoretical physics. In: Lecture Notes in Physics, Vol. 80. dell'Antonio, G.F., et al. (eds.). Berlin, Heidelberg, New York: Springer 1978

47. Seiler, E., Simon, B. : Ann. Phys. (N.Y.) 97, 470 (1976)

48. Seiler, E., Stamatescu, I.O., Zwanziger, D.: In preparation 
49. Simon, B.: Trace ideals and their applications. Cambridge: Cambridge University Press 1979

50. Simon, B.: Functional integration and quantum physics. New York: Academic Press 1979

51. Steinhardt, P.J. : Particle physics and the new cosmology. University of Pennsylvania preprint 1982 Albrecht, A., Steinhardt, P.J.: Phys. Rev. Lett. 48, 1220 (1982)

52. Susskind, L.: Phys. Rev. D 20, 2610 (1979)

53. Svetitsky, B., Yaffe, L.G.: Phys. Rev. D 26, 963 (1982); Nucl. Phys. B 210 [FS6], 423 (1982)

54. 't Hooft, G.: In: Recent developments in gauge theories. 't Hooft, G., et al. (eds.). New York: Plenum Press 1980

55. Tomboulis, E.T.: Phys. Rev. Lett. 50, 885 (1983)

56. Varadarajan, V.S.: Lie groups, Lie algebras and their representations. Englewood Cliffs, NJ: Prentice Hall 1974

57. Villain, J.: J. Physique 36, 581 (1975)

58. Wallace, A.P.: Algebraic topology, homology and cohomology. New York: Benjamin 1970

59. Wegner, F.: J. Math. Phys. 12, 2259 (1971)

60. Weiss, N.: Phys. Rev. D 24, 475 (1981); D 25, 2667 (1982)

61. Wilson, K.: Phys. Rev. D 10, 2445 (1974)

Communicated by G. Mack

Received May 30, 1983; in revised form June 13, 1983 